\title{
Ligand and Base Free Copper(II)-Catalyzed C-N Bond Formation: Cross-coupling Reactions of Organoboron Compounds with Aliphatic Amines and Anilines
}

\author{
Tan D. Quach and Robert A. Batey.* \\ Davenport Research Laboratories, Department of Chemistry, University of Toronto, \\ 80 St. George Street, Toronto, Ontario, M5S 3H6, Canada \\ rbatey@chem.utoronto.ca
}

\section{General Synthetic Methods}

$\mathrm{CH}_{2} \mathrm{Cl}_{2}$ was distilled from $\mathrm{CaH}_{2}$ under argon. All other commercial solvents and reagents were used as received from the Aldrich Chemical Company, Fischer Scientific Ltd., EMD Chemicals Inc., Strem or BDH. The $4 \AA$ molecular sieves were purchased through the Aldrich Chemical Company in powdered form, activated and stored in a oven at $210{ }^{\circ} \mathrm{C}$ until their use. All glassware was oven dried at $210^{\circ} \mathrm{C}$ and allowed to cool under a stream of dry oxygen. The oxygen gas (UHP/zero grade) was purchased from Air Products and was dried through a tube of Drierite (anhydrous $\mathrm{CaSO}_{4}$ ) prior to connection to a manifold for delivery into the reaction flask.

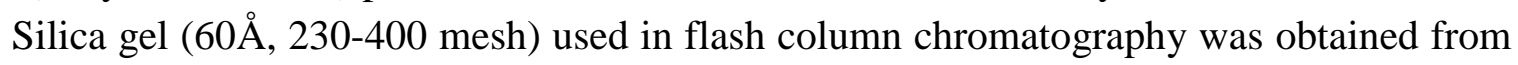
Silicycle and was used as received. Analytical thin-layer chromatography (TLC) was performed on pre-coated silica gel plates (Ultra Pure Silica Gel Plates purchased from Silicycle), visualized with a Spectroline $\mathrm{UV}_{254}$ lamp, and stained with a $20 \%$ phosphomolybdic acid in ethanol solution. Solvent systems associated with $\mathrm{R}_{f}$ values and flash column chromatography are reported as $\mathrm{v} / \mathrm{v}$ ratios.

Melting points were obtained using a Fisher-Johns melting point apparatus and are uncorrected. Specific optical rotations were determined on a Perkin-Elmer 243B Polarimeter under the conditions indicated using the sodium D line $(589 \mathrm{~nm})$. Sample concentrations are reported as grams per $100 \mathrm{~mL}$ solvent. Analytical chiral HPLC (of 3f, 3n and 3s) was performed on a Chiralcel OD column $(4.6$ x $250 \mathrm{~mm})$ obtained from Daicel Chemical Industries, Ltd. ${ }^{1} \mathrm{H}$ and ${ }^{13} \mathrm{C}$ NMR were recorded at $300 \mathrm{MHz}$ and $75 \mathrm{MHz}$ respectively on a Varian Gemini 300 or Mercury 300 spectrometer. Proton chemical shifts were internally referenced to the residual proton resonance in $\mathrm{CDCl}_{3}(\delta 7.26 \mathrm{ppm})$. Carbon chemical shifts were internally referenced to the deuterated solvent signals in $\mathrm{CDCl}_{3}(\delta 77.20 \mathrm{ppm})$. FT-IR spectra were recorded on a PerkinElmer Spectrum 1000 spectrometer with samples loaded as neat films on $\mathrm{NaCl}$ plates. Low and high resolution mass spectra were recorded on a Bell and Howell 21-490 spectrometer and an AEI MS3074 spectrometer respectively.

References following compound names indicate literature articles where ${ }^{1} \mathrm{H}$ and ${ }^{13} \mathrm{C}$ NMR data have previously been reported. 


\section{Synthetic Methods and Characterization Data}

\section{Representative Procedure for the Cross-Coupling of $\mathrm{PhBF}_{3}{ }^{-} \mathrm{K}^{+}$with Aliphatic Amines}

A suspension of $\mathrm{PhBF}_{3}{ }^{-} \mathrm{K}^{+}(0.368 \mathrm{~g}, 2.00 \mathrm{mmol}), \mathrm{Cu}(\mathrm{OAc})_{2} \cdot \mathrm{H}_{2} \mathrm{O}(20.0 \mathrm{mg}, 0.100 \mathrm{mmol})$, and powdered $4 \AA$ molecular sieves $(0.750 \mathrm{~g})$ in $\mathrm{CH}_{2} \mathrm{Cl}_{2}(8.00 \mathrm{~mL})$ was stirred for 5 minutes at room temperature. To this stirring suspension was added $n$-butylamine $(0.072 \mathrm{~g}, 99.0 \mu \mathrm{L}, 1.00 \mathrm{mmol})$. The reaction mixture was then sealed with a rubber septa (heated if necessary), and stirred under an atmosphere of $\mathrm{O}_{2}$. Following a period of $24 \mathrm{~h}$, the crude reaction mixture was filtered through a plug of celite to remove the molecular sieves and any insoluble byproducts and then concentrated in vacuo to afford the crude product mixture. The product (3a) was isolated by silica gel column chromatography (eluting with hexanes:EtOAc 9:1 3:1 gradient) as a pale yellow oil in $89 \%$ yield $(0.133 \mathrm{~g}, 0.89 \mathrm{mmol})$.

\section{Representative Procedure for the Cross-Coupling of $\mathrm{PhB}(\mathrm{OH})_{2}$ with Aliphatic Amines}

The above procedure was followed with substitution of $\mathrm{PhB}(\mathrm{OH})_{2}(0.244 \mathrm{~g}, 2.00 \mathrm{mmol})$ in place of the potassium organotrifluoroborate salt. The product (3a) was isolated as a pale yellow oil in $92 \%$ yield $(0.137 \mathrm{~g}, 0.89 \mathrm{mmol})$.

\section{Representative Procedure for the Activation of Amine Hydrohalide Salts and Subsequent Cross-Coupling with $\mathrm{PhB}(\mathrm{OH})_{2}$}

To a stirring solution of 3-bromopropylamine hydrobromide $(0.219 \mathrm{~g}, 1.00 \mathrm{~mL})$ in acetonitrile $(10.0 \mathrm{~mL})$ was added Amberlyst A-21 resin $(0.750 \mathrm{~g})$. The suspension was stirred at room temperature for $30 \mathrm{~min}$, then filtered and washed with $\mathrm{CH}_{2} \mathrm{Cl}_{2}$ to remove the resin. The filtrant was collected and concentrated in vacuo. The residue was then redissolved in acetonitrile (1.00 $\mathrm{mL})$ and added to a prestirring suspension of $\mathrm{PhB}(\mathrm{OH})_{2}(0.244 \mathrm{~g}, 2.00 \mathrm{mmol}), \mathrm{Cu}(\mathrm{OAc})_{2} \cdot \mathrm{H}_{2} \mathrm{O}$ (20.0 mg, $0.100 \mathrm{mmol}$ ), and powdered $4 \AA$ molecular sieves $(0.750 \mathrm{~g})$ in $\mathrm{CH}_{2} \mathrm{Cl}_{2}(8.00 \mathrm{~mL})$. The reaction mixture was then sealed with a rubber septa, heated to $40{ }^{\circ} \mathrm{C}$, and stirred under an atmosphere of $\mathrm{O}_{2}$. Following a period of $24 \mathrm{~h}$, the crude reaction mixture was filtered through a plug of celite to remove the molecular sieves and any insoluble byproducts, and then concentrated in vacuo to afford the crude product mixture. The product (3l) was isolated by silica gel column chromatography (eluting with hexanes:EtOAc 9:1 3:1 gradient) as a clear oil in $86 \%$ yield $(0.184 \mathrm{~g}, 0.860 \mathrm{mmol})$. 


\section{Products of Experiments Summarized in Table 1}

n-Butylphenylamine (Alberti, A.; Cane, F.; Dembech, P.; Lazzari, D.; Ricci, A.; Seconi, G. $J$. Org. Chem. 1996, 61, 1677-1681.)

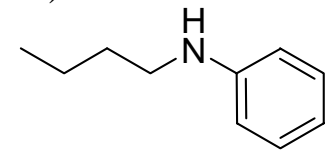

3a

Reactions were performed at room temperature. Product was isolated as a pale yellow oil in $89 \%$ yield from $\mathrm{PhBF}_{3}{ }^{-} \mathrm{K}^{+}$and in $92 \%$ yield from $\mathrm{PhB}(\mathrm{OH})_{2}: \mathrm{R}_{f}=0.80$ (3:1 hexanes:EtOAc); ${ }^{1} \mathrm{H}$ NMR $\left(300 \mathrm{MHz}, \mathrm{CDCl}_{3}\right) \delta 7.22(2 \mathrm{H}, \mathrm{ddd}, J=8.5,7.5,1.0 \mathrm{~Hz}), 6.74(1 \mathrm{H}, \mathrm{tt}, J=7.5,1.0 \mathrm{~Hz})$, $6.65(2 \mathrm{H}, \mathrm{dd}, J=8.5,1.0 \mathrm{~Hz}), 3.62(1 \mathrm{H}, \mathrm{br} \mathrm{s}), 3.15(2 \mathrm{H}, \mathrm{tt}, J=7.0 \mathrm{~Hz}), 1.65(2 \mathrm{H}$, quintet, $J=7.0$ $\mathrm{Hz}), 1.48(2 \mathrm{H}$, sextet, $J=7.0 \mathrm{~Hz}), 1.01(3 \mathrm{H}, \mathrm{t}, J=7.5 \mathrm{~Hz}) ;{ }^{13} \mathrm{C} \mathrm{NMR}\left(75 \mathrm{MHz}, \mathrm{CDCl}_{3}\right)$ $\delta 148.70,129.35,117.19,112.81,43.80,31.83,20.48,14.06$.

Isopropylphenylamine (Shaffer, C. L.; Morton, M. D.; Hanzlik, R. P. J. Am. Chem. Soc. 2001, $123,8502-8508$.

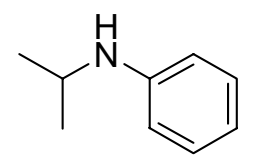

3b

Reactions were performed at room temperature. Product was isolated as a clear oil in $98 \%$ yield from both $\mathrm{PhBF}_{3}{ }^{-} \mathrm{K}^{+}$and $\mathrm{PhB}(\mathrm{OH})_{2}: \mathrm{R}_{f}=0.70$ (9:1 hexanes:EtOAc); ${ }^{1} \mathrm{H}$ NMR (300 MHz, $\left.\mathrm{CDCl}_{3}\right) \delta 7.25(2 \mathrm{H}, \mathrm{ddd}, J=8.0,7.5,1.0 \mathrm{~Hz}), 6.75(1 \mathrm{H}, \mathrm{tt}, J=7.5,1.0 \mathrm{~Hz}), 6.66(2 \mathrm{H}, \mathrm{dd}, J=8.0$, $1.0 \mathrm{~Hz}), 3.71(1 \mathrm{H}$, septet, $J=6.5 \mathrm{~Hz}), 3.50(1 \mathrm{H}$, br s$), 1.28(6 \mathrm{H}, \mathrm{d}, J=6.5 \mathrm{~Hz}) ;{ }^{13} \mathrm{C}$ NMR $(75$ $\left.\mathrm{MHz}, \mathrm{CDCl}_{3}\right) \delta 147.64,129.40,117.05,113.34,44.28,23.14$.

Cyclohexylphenylamine (Kawakami, T.; Sugimoto, T.; Shibata, I.; Baba, A.; Matsuda, H.; Sonoda, N. J. Org. Chem. 1995, 60, 2677-2682.)

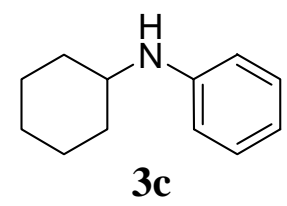

Product was isolated as a clear oil in $79 \%$ yield from $\mathrm{PhBF}_{3}{ }^{-} \mathrm{K}^{+}$at room temperature; and in $85 \%$ yield from $\mathrm{PhB}(\mathrm{OH})_{2}$ at $40{ }^{\circ} \mathrm{C}: \mathrm{R}_{f}=0.60$ (3:1 hexanes:EtOAc); ${ }^{1} \mathrm{H}$ NMR $\left(300 \mathrm{MHz}, \mathrm{CDCl}_{3}\right)$ $\delta 7.17(2 \mathrm{H}, \mathrm{ddd}, J=8.5,7.5,2.0 \mathrm{~Hz}), 6.67(1 \mathrm{H}, \mathrm{tt}, J=7.5,1.0 \mathrm{~Hz}), 6.61(2 \mathrm{H}$, ddd, $J=8.5,2.0$, $1.0 \mathrm{~Hz}$ ), $3.53(1 \mathrm{H}$, br s), 3.27 (1H, dddd, $J=14.0,7.5,6.5,4.0 \mathrm{~Hz}), 2.14-2.02(2 \mathrm{H}, \mathrm{m}), 1.84$ $1.62(3 \mathrm{H}, \mathrm{m}), 1.48-1.10(5 \mathrm{H}, \mathrm{m}) ;{ }^{13} \mathrm{C} \mathrm{NMR}\left(75 \mathrm{MHz}, \mathrm{CDCl}_{3}\right) \delta 147.59,129.43,116.99,113.30$, $51.86,33.68,26.13,25.21$. 
t-Butylphenylamine (Arnauld, T.; Barton, D. H. R.; Doris, E. Tetrahedron 1997, 53, 41374144.)

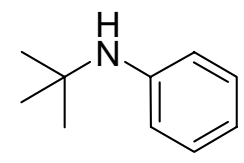

3d

Product was isolated as a light beige oil in $26 \%$ yield from $\mathrm{PhBF}_{3}{ }^{-} \mathrm{K}^{+}$at room temperature; and in $39 \%$ yield from $\mathrm{PhB}(\mathrm{OH})_{2}$ at $40{ }^{\circ} \mathrm{C}: \mathrm{R}_{f}=0.40$ (9:1 hexanes:EtOAc); ${ }^{1} \mathrm{H}$ NMR $(300 \mathrm{MHz}$, $\left.\mathrm{CDCl}_{3}\right) \delta 7.16(2 \mathrm{H}, \mathrm{t}, J=7.5 \mathrm{~Hz}), 6.8-6.72(3 \mathrm{H}, \mathrm{m}), 3.34(1 \mathrm{H}, \mathrm{br} \mathrm{s}), 1.34(9 \mathrm{H}, \mathrm{s}) ;{ }^{13} \mathrm{C} \mathrm{NMR}(75$ $\left.\mathrm{MHz} \mathrm{CDCl}_{3}\right) \delta 147.06,129.08,118.49,117.68,51.66,30.28$.

Adamantan-1-ylphenylamine (Olifirov, D. I.; Koshchii, V. A.; Kozlikovskii, Y. B. J. Org. Chem. USSR (Engl. Transl.), 1992, 28, 152-157.)

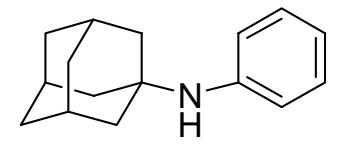

$3 \mathbf{e}$

Reactions were performed at $40{ }^{\circ} \mathrm{C}$. Product was isolated as a pale beige solid in $57 \%$ yield from $\mathrm{PhBF}_{3}{ }^{-} \mathrm{K}^{+}$and in $67 \%$ yield from $\mathrm{PhB}(\mathrm{OH})_{2}: \mathrm{R}_{f}=0.60$ (3:1 hexanes:EtOAc); ${ }^{1} \mathrm{H}$ NMR (300 $\left.\mathrm{MHz}, \mathrm{CDCl}_{3}\right) \delta 7.19$ - $7.13(2 \mathrm{H}, \mathrm{m}), 6.83$ - $6.77(3 \mathrm{H}, \mathrm{m}), 3.31(1 \mathrm{H}, \mathrm{br} \mathrm{s}), 2.12(3 \mathrm{H}, \mathrm{m}), 1.88(6 \mathrm{H}$, $\mathrm{d}, J=3.0 \mathrm{~Hz}), 1.77-1.71(6 \mathrm{H}, \mathrm{m}) ;{ }^{13} \mathrm{C} \mathrm{NMR}\left(100 \mathrm{MHz}, \mathrm{CDCl}_{3}\right) \delta 146.21,128.89,119.25$, 119.19, 52.35, 43.62, 36.63, 29.87; LRMS (EI): $m / z$ (rel. intensity) = 229 (9), 227 (51), 171 (15), 170 (100), 135 (41), 133 (9), 107 (5), 94 (5), 93 (21), 92 (11), 91 (7), 79 (14), 77 (12), 67 (6); HRMS (EI): $m / z$ calcd. for $\left(\mathrm{M}^{+}\right)=227.1674$, found $=227.1673$.

S-(-)-N-(1-Phenylethyl)aniline (Landor, S. R.; Sonola, O. O.; Tatchell, A. R. Bull. Chem. Soc. Jpn. 1984, 57, 1658-1661.)

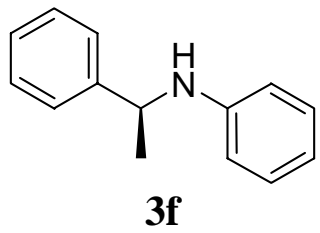

Product was isolated as a clear oil in $91 \%$ yield from $\mathrm{PhBF}_{3}{ }^{-} \mathrm{K}^{+}$at room temperature: $\mathrm{R}_{f}=0.44$ (9:1 hexanes:EtOAc $) ;[\alpha]_{\mathrm{D}}{ }^{22}=-5.15^{\circ}\left(\mathrm{c}=10, \mathrm{CHCl}_{3}\right) ;{ }^{1} \mathrm{H}$ NMR $\left(300 \mathrm{MHz}, \mathrm{CDCl}_{3}\right) \delta 7.37$ $7.15(5 \mathrm{H}, \mathrm{m}), 7.07(2 \mathrm{H}, \mathrm{t}, J=7.5 \mathrm{~Hz}), 6.63(1 \mathrm{H}, \mathrm{t}, J=7.5 \mathrm{~Hz}), 6.49(2 \mathrm{H}, \mathrm{d}, J=7.5 \mathrm{~Hz}), 4.46$ $(1 \mathrm{H}, \mathrm{q}, J=7.0 \mathrm{~Hz}), 3.98(1 \mathrm{H}$, br s$), 1.48(3 \mathrm{H}, \mathrm{d}, J=7.0 \mathrm{~Hz}) ;{ }^{13} \mathrm{C}$ NMR $\left(75 \mathrm{MHz}, \mathrm{CDCl}_{3}\right)$ $\delta 147.42,145.37,129.24,128.77,127.00,125.98,117.36,113.43,53.57,25.16$. 


\section{[2-(3,4-Dimethoxyphenyl)ethyl]phenylamine}

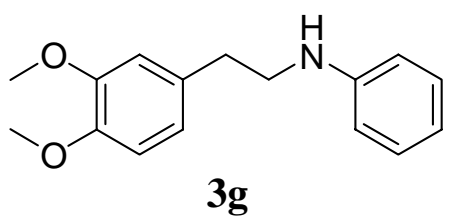

Product was isolated as a clear oil in $90 \%$ yield from $\mathrm{PhBF}_{3}{ }^{-} \mathrm{K}^{+}$at room temperature; and in $95 \%$ yield from $\mathrm{PhB}(\mathrm{OH})_{2}$ at $40{ }^{\circ} \mathrm{C}: \mathrm{R}_{f}=0.70$ (1:1 hexanes:EtOAc); ${ }^{1} \mathrm{H}$ NMR $\left(300 \mathrm{MHz}, \mathrm{CDCl}_{3}\right.$ ) $\delta 7.20(2 \mathrm{H}, \mathrm{ddd}, J=8.0,7.5,1.0 \mathrm{~Hz}), 6.86-6.70(4 \mathrm{H}, \mathrm{m}), 6.63(2 \mathrm{H}, \mathrm{dd}, J=7.5,1.0 \mathrm{~Hz}), 3.88$ $(3 \mathrm{H}, \mathrm{s}), 3.87(3 \mathrm{H}, \mathrm{s}), 3.68(1 \mathrm{H}, \mathrm{br} \mathrm{s}), 3.40(2 \mathrm{H}, \mathrm{t}, J=7.0 \mathrm{~Hz}), 2.88(2 \mathrm{H}, \mathrm{t}, J=7.0 \mathrm{~Hz}) ;{ }^{13} \mathrm{C} \mathrm{NMR}$ $\left(75 \mathrm{MHz}, \mathrm{CDCl}_{3}\right) \delta 149.13,148.15,147.78,131.94,129.39,120.80,117.59,113.16,112.17$, 111.53, 56.06, 55.96, 45.23, 35.13; IR (film) v 3394, 2932, 2360, 1603, 1513, 1465, 1419, 1319, 1261, 1235, 1156, 1140, 1027, 809, 750, $693 \mathrm{~cm}^{-1}$; LRMS (EI): $\mathrm{m} / z$ (rel. intensity) = $257(12)$, 152 (23), 151 (8), 107 (12), 106 (100), 86 (7), 84 (11), 79 (5), 77 (15); HRMS (EI): m/z calcd. for $\left(\mathrm{M}^{+}\right)=257.1416$, found $=257.1418$.

\section{(2-Methoxyethyl)phenylamine}

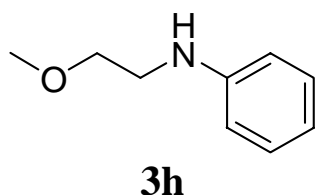

Product was isolated as a clear oil in $32 \%$ yield from $\mathrm{PhBF}_{3}{ }^{-} \mathrm{K}^{+}$at room temperature; and in $79 \%$ yield from $\mathrm{PhBF}_{3}{ }^{-} \mathrm{K}^{+}$and $94 \%$ yield from $\mathrm{PhB}(\mathrm{OH})_{2}$ at $40{ }^{\circ} \mathrm{C}: \mathrm{R}_{f}=0.40$ (3:1 hexanes:EtOAc); ${ }^{1} \mathrm{H}$ NMR $\left(300 \mathrm{MHz}, \mathrm{CDCl}_{3}\right) \delta 7.19(2 \mathrm{H}, \mathrm{t}, J=7.5 \mathrm{~Hz}), 6.70(1 \mathrm{H}, \mathrm{t}, J=7.5 \mathrm{~Hz}), 6.61(2 \mathrm{H}, \mathrm{d}, J=$ $7.5 \mathrm{~Hz}), 4.02\left(1 \mathrm{H}\right.$, br s), $3.57(2 \mathrm{H}, \mathrm{t}, J=5.0 \mathrm{~Hz}), 3.37(3 \mathrm{H}, \mathrm{s}), 3.26(2 \mathrm{H}, \mathrm{t}, J=5.0 \mathrm{~Hz}) ;{ }^{13} \mathrm{C} \mathrm{NMR}$ $\left(75 \mathrm{MHz}, \mathrm{CDCl}_{3}\right) \delta 148.31,129.31,117.67,113.17,71.09,58.81,43.53$; IR (film) v 3051, 2925, 2891, 1603, 1506, 1472, 1319, 1259, 1193, 1117, 1071, 1025, 871, 750, $693 \mathrm{~cm}^{-1}$; LRMS (EI): $\mathrm{m} / \mathrm{z}($ rel. intensity) = 152 (9), 151 (48), 107 (14), 106 (100), 94 (7), 79 (12), 78 (5), 77 (24), 65 (7), 51 (11); HRMS (EI): $\mathrm{m} / z$ calcd. for $\left(\mathrm{M}^{+}\right)=151.0997$, found $=151.0991$.

\section{(3-Methoxypropyl)phenylamine}

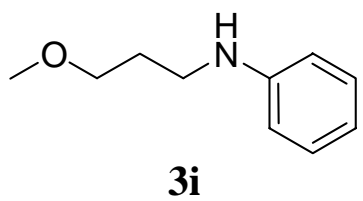

Product was isolated as a clear oil in $78 \%$ yield from $\mathrm{PhBF}_{3}{ }^{-} \mathrm{K}^{+}$at room temperature; and in $85 \%$ yield from $\mathrm{PhB}(\mathrm{OH})_{2}$ at $40{ }^{\circ} \mathrm{C}: \mathrm{R}_{f}=0.50$ (3:1 hexanes:EtOAc); ${ }^{1} \mathrm{H}$ NMR (300 $\mathrm{MHz}, \mathrm{CDCl}_{3}$ ) $\delta 7.19(2 \mathrm{H}, \mathrm{ddd}, J=7.5,3.0,1.0 \mathrm{~Hz}), 6.70(1 \mathrm{H}, \mathrm{tt}, J=7.5,1.0 \mathrm{~Hz}), 6.63(2 \mathrm{H}, \mathrm{dd}, J=7.5,1.0$ $\mathrm{Hz}), 3.94(1 \mathrm{H}, \mathrm{br} \mathrm{s}), 3.53(2 \mathrm{H}, \mathrm{t}, J=6.0 \mathrm{~Hz}), 3.37(3 \mathrm{H}, \mathrm{s}), 3.24(2 \mathrm{H}, \mathrm{t}, J=6.0 \mathrm{~Hz}), 1.90(2 \mathrm{H}$, quintet, $J=6.0 \mathrm{~Hz}) ;{ }^{13} \mathrm{C} \mathrm{NMR}\left(75 \mathrm{MHz}, \mathrm{CDCl}_{3}\right) \delta 148.66,129.37,117.27,112.87,71.37,58.88$, 41.92, 29.54; IR (film) v 3394, 3051, 3021, 2924, 2871, 1604, 1508, 1478, 1433, 1389, 1321, 
1262, 1180, 1117, 749, $693 \mathrm{~cm}^{-1}$; LRMS (EI): $\mathrm{m} / \mathrm{z}$ (rel. intensity) = 166 (6), 165 (37), 107 (13), 106 (100), 104 (6), 93 (11), 79 (10), 77 (18), 65 (6), 51 (7); HRMS (EI): m/z calcd. for $\left(\mathrm{M}^{+}\right)=$ 165.1154 , found $=165.1150$.

(3,7-Dimethylocta-2,6-dienyl)phenylamine (Ranu, B. C.; Majee, A.; Sarkar, A. J. Org. Chem. 1998, 63, 370-373.)

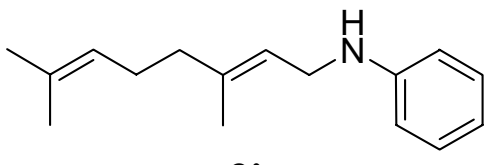

$3 \mathbf{j}$

Reactions were performed at $40{ }^{\circ} \mathrm{C}$. Product was isolated as a yellow oil in $80 \%$ yield from $\mathrm{PhBF}_{3}{ }^{-} \mathrm{K}^{+}$and in $89 \%$ yield from $\mathrm{PhB}(\mathrm{OH})_{2}: \mathrm{R}_{f}=0.75$ (3:1 hexanes:EtOAc); ${ }^{1} \mathrm{H}$ NMR (300 $\left.\mathrm{MHz} \mathrm{CDCl}_{3}\right) \delta 7.29(2 \mathrm{H}, \mathrm{ddd}, J=8.5,7.5,2.0 \mathrm{~Hz}), 6.82(1 \mathrm{H}, \mathrm{tt}, J=7.5,1.0 \mathrm{~Hz}), 6.72(2 \mathrm{H}$, ddd, $J=8.5,2.0,1.0 \mathrm{~Hz}), 5.49-5.41(1 \mathrm{H}, \mathrm{m}), 5.25-5.18(1 \mathrm{H}, \mathrm{m}), 3.81(2 \mathrm{H}, \mathrm{d}, J=6.5 \mathrm{~Hz}), 3.67(1 \mathrm{H}$, br s), $2.28-2.14(4 \mathrm{H}, \mathrm{m}), 1.82(3 \mathrm{H}, \mathrm{s}), 1.81(3 \mathrm{H}, \mathrm{s}), 1.73(3 \mathrm{H}, \mathrm{s}) ;{ }^{13} \mathrm{C}$ NMR $\left(100 \mathrm{MHz}, \mathrm{CDCl}_{3}\right)$ $\delta 148.58,139.10,131.81,129.33,124.10,121.70,117.40,113.02$, 42.10, 39.67, 26.59, 25.86, $17.86,16.51$.

\section{Phenyl(tetrahydrofuran-2-ylmethyl)amine}

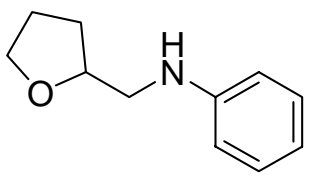

3k

Reactions were performed at $40{ }^{\circ} \mathrm{C}$. Product was isolated as a pale yellow oil in $83 \%$ yield from $\mathrm{PhBF}_{3}{ }^{-} \mathrm{K}^{+}$and in $91 \%$ yield from $\mathrm{PhB}(\mathrm{OH})_{2}: \mathrm{R}_{f}=0.33$ (3:1 hexanes:EtOAc); ${ }^{1} \mathrm{H}$ NMR (300 $\left.\mathrm{MHz}, \mathrm{CDCl}_{3}\right) \delta 7.20(2 \mathrm{H}$, ddd, $J=8.5,7.5,2.0 \mathrm{~Hz}), 6.74(1 \mathrm{H}, \mathrm{tt}, J=7.5,1.0 \mathrm{~Hz}), 6.66$ (2H, ddd, $J=8.5,2.0,1.0 \mathrm{~Hz}), 4.15(1 \mathrm{H}, \mathrm{dddd}, J=7.0,7.0,7.0,4.0 \mathrm{~Hz}), 4.05(1 \mathrm{H}, \mathrm{br} \mathrm{s}), 3.92(1 \mathrm{H}, \mathrm{ddd}, J=$ 8.0, 7.0, 7.0 Hz), $3.81(1 \mathrm{H}, \mathrm{ddd}, J=8.0,7.0,7.0 \mathrm{~Hz}), 3.28(1 \mathrm{H}, \mathrm{dd}, J=12.5,4.0 \mathrm{~Hz}), 3.10(1 \mathrm{H}$, $\mathrm{dd}, J=12.5,7.5 \mathrm{~Hz}), 2.11-1.90(3 \mathrm{H}, \mathrm{m}), 1.74-1.62(1 \mathrm{H}, \mathrm{m}) ;{ }^{13} \mathrm{C} \mathrm{NMR}\left(100 \mathrm{MHz}, \mathrm{CDCl}_{3}\right)$ $\delta 148.50,129.28,117.53,113.10,77.64,68.12,48.24,29.19,25.89$; IR (film) v 3390, 3051, 3021, 2971, 2869, 1603, 1506, 1459, 1433, 1378, 1360, 1320, 1256, 1180, 1070, 749, $693 \mathrm{~cm}^{-1}$; LRMS (EI): $m / z$ (rel. intensity) = 178 (7), 177 (27), 107 (17), 106 (100), 79 (7), 77 (16), 71 (9), 50 (6); HRMS (EI): $\mathrm{m} / z$ calcd. for $\left(\mathrm{M}^{+}\right)=177.1154$, found $=177.1156$.

\section{(3-Bromopropyl)phenyl amine}

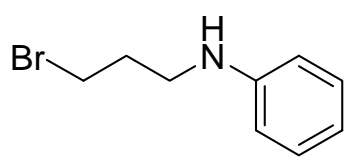

31

The amine hydrobromide salt was pretreated with Amberlyst A-21 resin. Reaction was performed at $40{ }^{\circ} \mathrm{C}$. Product was isolated as a clear oil in $86 \%$ yield from $\mathrm{PhB}(\mathrm{OH})_{2}$ over both 
steps: $\mathrm{R}_{f}=0.30$ (9:1 hexanes:EtOAc); ${ }^{1} \mathrm{H}$ NMR $\left(300 \mathrm{MHz}, \mathrm{CDCl}_{3}\right) \delta 7.29(2 \mathrm{H}, \mathrm{ddd}, J=7.5,2.0$, $1.5 \mathrm{~Hz}), 6.83(1 \mathrm{H}, \mathrm{tt}, J=7.5,1.5 \mathrm{~Hz}), 6.73(2 \mathrm{H}, \mathrm{dd}, J=7.5,2.0 \mathrm{~Hz}), 3.95(1 \mathrm{H}, \mathrm{br} \mathrm{s}), 3.60(2 \mathrm{H}, \mathrm{t}$, $J=6.5 \mathrm{~Hz}), 3.42(2 \mathrm{H}, \mathrm{t}, J=6.5 \mathrm{~Hz}), 2.24(2 \mathrm{H}$, quintet, $J=6.5 \mathrm{~Hz}) ;{ }^{13} \mathrm{C} \mathrm{NMR}\left(100 \mathrm{MHz}, \mathrm{CDCl}_{3}\right)$ $\delta$ 147.82, 129.46, 117.77, 113.01, 42.14, 32.07, 31.37; IR (film) v 3410, 3051, 3021, 2961, 1603, 1507, 1431, 1322, 1255, 1180, 1100, 991, 870, 751, $693 \mathrm{~cm}^{-1}$; LRMS (EI): $\mathrm{m} / z$ (rel. intensity) $=$ 215 (19), 213 (18), 107 (15), 106 (100), 104 (7), 79 (11), 78 (7), 77 (27), 65 (8); HRMS (EI): m/z calcd. for $\left(\mathrm{M}^{+}\right)=213.0153$, found $=213.0151$.

$N$-Phenyl-glycine ethyl ester (Anderson, W. K.; Bhattacharjee, D.; Houston, D. M.; J. Med. Chem. 1989, 32, 119-127.)

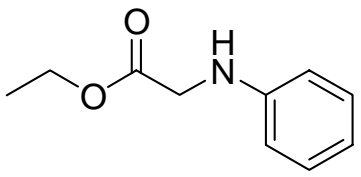

$3 m$

The amine hydrochloride salt was pretreated with Amberlyst A-21 resin. Reaction was performed at $40{ }^{\circ} \mathrm{C}$. Product was isolated as a clear crystalline solid in $84 \%$ yield from $\mathrm{PhB}(\mathrm{OH})_{2}$ over both steps: $\mathrm{R}_{f}=0.50$ (9:1 hexanes:EtOAc); ${ }^{1} \mathrm{H}$ NMR $\left(300 \mathrm{MHz}, \mathrm{CDCl}_{3}\right) \delta 7.21$ $(2 \mathrm{H}, \mathrm{t}, J=7.5 \mathrm{~Hz}), 6.76(1 \mathrm{H}, \mathrm{dt}, J=7.5,1.0 \mathrm{~Hz}), 6.62(2 \mathrm{H}, \mathrm{dd}, J=7.5,1.0 \mathrm{~Hz}), 4.28(1 \mathrm{H}, \mathrm{br} \mathrm{s})$, $4.25(2 \mathrm{H}, \mathrm{q}, J=7.0 \mathrm{~Hz}), 3.91(2 \mathrm{H}, \mathrm{s}), 1.31(3 \mathrm{H}, \mathrm{t}, J=7.0 \mathrm{~Hz}) ;{ }^{13} \mathrm{C} \mathrm{NMR}\left(100 \mathrm{MHz}, \mathrm{CDCl}_{3}\right)$ $\delta 171.33,147.20,129.48,118.37,113.19,61.49,46.05,14.36$.

S- $N$-Phenyl-phenylalanine methyl ester (Tunge, J. A.; Gately, D. A.; Norton, J. R. J. Amer. Chem. Soc. 1999, 121, 4520-4521.)

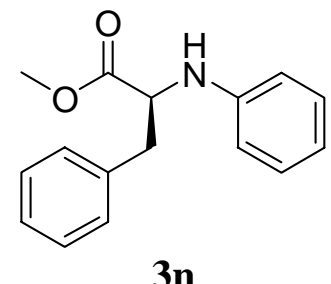

The amine hydrochloride salt was pretreated with Amberlyst A-21 resin. Reaction was performed at $40{ }^{\circ} \mathrm{C}$. Product was isolated as a light beige oil in $90 \%$ yield from $\mathrm{PhB}(\mathrm{OH})_{2}$ over both steps: $\mathrm{R}_{f}=0.40\left(3: 1\right.$ hexanes:EtOAc); $[\alpha]_{\mathrm{D}}{ }^{22}=+25.88^{\circ}\left(\mathrm{c}=10, \mathrm{CH}_{2} \mathrm{Cl}_{2}\right)$ for $>99 \%$ ee (Chiralcel OD, $10 \%$ ethanol/hexanes, $1.0 \mathrm{~mL} / \mathrm{min}$, retention times for $\mathrm{R}$ and $\mathrm{S}$ enantiomers are 7.16 and $9.78 \mathrm{~min}$ respectively) with determination of the absolute stereochemistry by comparison of the specific rotation with the literature value; ${ }^{1} \mathrm{H} \mathrm{NMR}\left(300 \mathrm{MHz}, \mathrm{CDCl}_{3}\right) \delta 7.48$ - $7.28(7 \mathrm{H}, \mathrm{m}), 6.90(1 \mathrm{H}, \mathrm{t}, J=7.5 \mathrm{~Hz}), 6.75(2 \mathrm{H}, \mathrm{d}, J=7.5 \mathrm{~Hz}), 4.53(1 \mathrm{H}, \mathrm{t}, J=6.5 \mathrm{~Hz}), 4.36$ $\left(1 \mathrm{H}\right.$, br s), $3.79(3 \mathrm{H}, \mathrm{s}), 3.28(2 \mathrm{H}$, ddd, $J=20.0,13.5,6.5 \mathrm{~Hz}) ;{ }^{13} \mathrm{C} \mathrm{NMR}\left(100 \mathrm{MHz}, \mathrm{CDCl}_{3}\right)$ $\delta 173.62,146.39,136.38,129.39,129.28,128.55,127.03,118.41,113.56,57.70,52.03,38.62$. 
N-Phenylpiperidine (Brenner, E.; Schneider, R.; Fort, Y.; Tetrahedron, 1999, 55, 1282912842.)

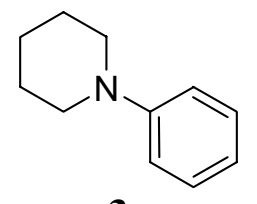

Product was isolated as a clear oil in $78 \%$ yield from $\mathrm{PhBF}_{3}{ }^{-} \mathrm{K}^{+}$at room temperature; and in $86 \%$ yield from $\mathrm{PhB}(\mathrm{OH})_{2}$ at $40{ }^{\circ} \mathrm{C}: \mathrm{R}_{f}=0.40$ (3:1 hexanes:EtOAc); ${ }^{1} \mathrm{H} \mathrm{NMR}\left(300 \mathrm{MHz}, \mathrm{CDCl}_{3}\right)$ $\delta 7.25(2 \mathrm{H}, \mathrm{dt}, J=7.5,1.0 \mathrm{~Hz}), 6.94(2 \mathrm{H}, \mathrm{dd}, J=7.5,1.0 \mathrm{~Hz}), 6.82(1 \mathrm{H}, \mathrm{dt}, J=7.5,1.0 \mathrm{~Hz}), 3.16$ $(4 \mathrm{H}, \mathrm{t}, J=5.5 \mathrm{~Hz}), 1.76-1.66(4 \mathrm{H}, \mathrm{m}), 1.62-1.53(2 \mathrm{H}, \mathrm{m}) ;{ }^{13} \mathrm{C}$ NMR $\left(75 \mathrm{MHz}, \mathrm{CDCl}_{3}\right)$ $\delta 152.47,129.19,119.36,116.72,50.87,26.07,24.52$.

4-Phenyl-morpholine (Ishikawa, T.; Uedo, E.; Tani, R.; Saito, S. J. Org. Chem. 2001, 66, 186191.)

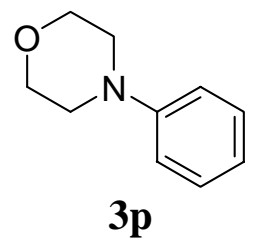

Reactions were performed at $40{ }^{\circ} \mathrm{C}$. Product was isolated as a pale light beige, crystalline solid in $81 \%$ yield from $\mathrm{PhBF}_{3}{ }^{-} \mathrm{K}^{+}$and in $90 \%$ yield from $\mathrm{PhB}(\mathrm{OH})_{2}: \mathrm{R}_{f}=0.45$ (3:1 hexanes:EtOAc); ${ }^{1} \mathrm{H}$ NMR $\left(300 \mathrm{MHz}, \mathrm{CDCl}_{3}\right) \delta 7.31(2 \mathrm{H}, \mathrm{ddd}, J=9.0,7.5,1.5 \mathrm{~Hz}), 6.96-6.88(3 \mathrm{H}, \mathrm{m}), 3.91-3.85$ $(4 \mathrm{H}, \mathrm{m}), 3.20-3.15(4 \mathrm{H}, \mathrm{m}) ;{ }^{13} \mathrm{C} \mathrm{NMR}\left(100 \mathrm{MHz}, \mathrm{CDCl}_{3}\right) \delta 151.41,129.33,120.17,115.83$, $67.08,49.48$.

\section{8-Phenyl-1,4-dioxa-8-azaspiro[4.5]decane}

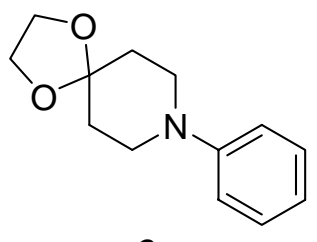

$3 \mathbf{q}$

Reactions were performed at $40{ }^{\circ} \mathrm{C}$. Product was isolated as a clear oil in $87 \%$ yield from $\mathrm{PhBF}_{3}{ }^{-}$ $\mathrm{K}^{+}$and in $86 \%$ yield from $\mathrm{PhB}(\mathrm{OH})_{2}: \mathrm{R}_{f}=0.40$ (3:1 hexanes:EtOAc); ${ }^{1} \mathrm{H}$ NMR $(300 \mathrm{MHz}$, $\left.\mathrm{CDCl}_{3}\right) \delta 7.34(2 \mathrm{H}$, ddd, $J=7.5,2.0,1.5 \mathrm{~Hz}), 7.04(2 \mathrm{H}, \mathrm{dd}, J=7.5,2.0 \mathrm{~Hz}), 6.93(1 \mathrm{H}, \mathrm{dt}, J=$ $7.5,1.5 \mathrm{~Hz}), 4.07(4 \mathrm{H}, \mathrm{s}), 3.42(4 \mathrm{H}, \mathrm{dd}, J=6.0,6.0 \mathrm{~Hz}), 1.94(4 \mathrm{H}, \mathrm{dd}, J=6.0,6.0 \mathrm{~Hz}) ;{ }^{13} \mathrm{C}$ NMR $\left(100 \mathrm{MHz}, \mathrm{CDCl}_{3}\right) \delta 151.08,129.19,119.49,116.70,107.27,64.41,47.83,34.64$; IR (film) v 2956, 2882, 2828, 1599, 1497, 1466, 1365, 1228, 1143, 1105, 1037, 962, 946, 929, 896, 757, 693, $529 \mathrm{~cm}^{-1}$; LRMS (EI): $\mathrm{m} / \mathrm{z}$ (rel. intensity) = 220 (18), 219 (100), 218 (10), 174 (28), 158 (20), 133 (18), 132 (55), 106 (11), 105 (73), 104 (25), 77 (21); HRMS (EI): m/z calcd. for $\left(\mathrm{M}^{+}\right)=219.1259$, found $=219.1265$. 
1-Phenyl-piperidin-4-one (Hermant, R. M.; Bakker, N. A. C.; Scherer, T.; Krijnen, B.; Verhoeven, J. W. J. Amer. Chem. Soc. 1990, 112, 1214-1221.)

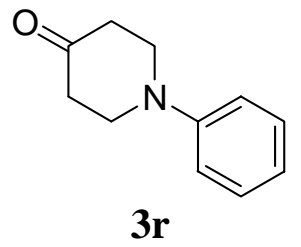

The amine hydrochloride salt was pretreated with Amberlyst A-21 resin. Reaction was performed at $40{ }^{\circ} \mathrm{C}$. Product was isolated as a light beige solid in $83 \%$ yield from $\mathrm{PhB}(\mathrm{OH})_{2}$ over both steps: $\mathrm{R}_{f}=0.33\left(3: 1\right.$ hexanes:EtOAc); ${ }^{1} \mathrm{H}$ NMR $\left(300 \mathrm{MHz}, \mathrm{CDCl}_{3}\right) \delta 7.29(2 \mathrm{H}, \mathrm{ddd}, J=7.5$, 2.0, $1.5 \mathrm{~Hz}), 6.97(2 \mathrm{H}, \mathrm{dd}, J=7.5,2.0 \mathrm{~Hz}), 6.91(1 \mathrm{H}, \mathrm{dt}, J=7.5,1.5 \mathrm{~Hz}), 3.60(4 \mathrm{H}, \mathrm{t}, J=6.0$ $\mathrm{Hz}), 2.54(4 \mathrm{H}, \mathrm{t}, J=6.0 \mathrm{~Hz}) ;{ }^{13} \mathrm{C} \mathrm{NMR}\left(100 \mathrm{MHz}, \mathrm{CDCl}_{3}\right) \delta 208.35,149.25,129.58,119.96$, $116.01,48.96,40.86$.

$N$-Phenyl-L-proline methyl ester (Ishikawa, T.; Uedo, E.; Tani, R.; Saito, S. J. Org. Chem. 2001, 66, 186-191.)

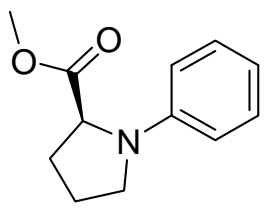

3s

The amine hydrochloride salt was pretreated with Amberlyst A-21 resin. Reaction was performed at $40{ }^{\circ} \mathrm{C}$. Product was isolated as a pale yellow oil in $74 \%$ yield from $\mathrm{PhB}(\mathrm{OH})_{2}$ over both steps: $\mathrm{R}_{f}=0.50\left(3: 1\right.$ hexanes:EtOAc); $[\alpha]_{\mathrm{D}}{ }^{22}=-13.81^{\circ}\left(\mathrm{c}=1.0, \mathrm{CHCl}_{3}\right)$ for $>99 \%$ ee (Chiralcel OD, $10 \%$ ethanol/hexanes, $1.0 \mathrm{~mL} / \mathrm{min}$, retention times for $\mathrm{R}$ and $\mathrm{S}$ enantiomers are 6.19 and $8.77 \mathrm{~min}$ respectively) with determination of the absolute stereochemistry by comparison of the specific rotation with the literature value; ${ }^{1} \mathrm{H}$ NMR $\left(300 \mathrm{MHz}, \mathrm{CDCl}_{3}\right) \delta 7.32$ $(2 \mathrm{H}$, ddd, $J=7.5,2.0,1.5 \mathrm{~Hz}), 6.81(1 \mathrm{H}, \mathrm{dt}, J=7.5,1.5 \mathrm{~Hz}), 6.64(2 \mathrm{H}, \mathrm{dd}, J=7.5,2.0 \mathrm{~Hz}), 4.34$ $(1 \mathrm{H}, \mathrm{dd}, J=8.0,2.0 \mathrm{~Hz}), 3.80(3 \mathrm{H}, \mathrm{s}), 3.70-3.66(1 \mathrm{H}, \mathrm{m}), 3.49-3.40(1 \mathrm{H}, \mathrm{m}), 2.45-2.07(4 \mathrm{H}$, $\mathrm{m}) ;{ }^{13} \mathrm{C} \mathrm{NMR}\left(100 \mathrm{MHz}, \mathrm{CDCl}_{3}\right) \delta 175.10,146.79,129.35,116.77,112.04,52.20,48.37,31.02$, 23.99 .

2-Phenyl-1,2,3,4-tetrahydroisoquinoline (Almena, J.; Foubelo, F.; Yus, M. Tetrahedron 1996, 52, 8545-8564.)<smiles>c1ccc(N2CCc3ccccc3C2)cc1</smiles>

$3 \mathbf{t}$

Reactions were performed at room temperature. Product was isolated as a clear oil in $72 \%$ yield from both $\mathrm{PhBF}_{3}{ }^{-} \mathrm{K}^{+}$and $\mathrm{PhB}(\mathrm{OH})_{2}: \mathrm{R}_{f}=0.50$ (9:1 hexanes:EtOAc); ${ }^{1} \mathrm{H}$ NMR (300 MHz, 
$\left.\mathrm{CDCl}_{3}\right) \delta 7.36-7.13(6 \mathrm{H}, \mathrm{m}), 7.02(2 \mathrm{H}, \mathrm{d}, J=7.5 \mathrm{~Hz}), 6.87(1 \mathrm{H}, \mathrm{t}, J=7.5 \mathrm{~Hz}), 4.45(2 \mathrm{H}, \mathrm{s})$, $3.60(2 \mathrm{H}, \mathrm{t}, J=6.0 \mathrm{~Hz}), 3.03(2 \mathrm{H}, \mathrm{t}, J=6.0 \mathrm{~Hz}) ;{ }^{13} \mathrm{C} \mathrm{NMR}\left(75 \mathrm{MHz}, \mathrm{CDCl}_{3}\right) \delta 150.44,134.41$, $129.16,128.98,128.48,126.51,126.30,126.00,118.66,115.16$.

1-Phenyl-pyrrole (Lee, C. K.; Jun, J. H.; Yu, J. S. J. Heterocycl. Chem. 2000, 37, 15-24.)

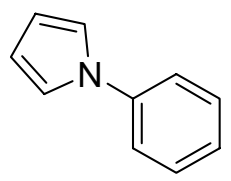

$3 \mathbf{u}$

Reactions were performed at $40{ }^{\circ} \mathrm{C}$. Product was isolated as a clear, crystalline solid in $80 \%$ yield from $\mathrm{PhBF}_{3}{ }^{-} \mathrm{K}^{+}$and in $89 \%$ yield from $\mathrm{PhB}(\mathrm{OH})_{2}$ : $\mathrm{R}_{f}=0.60$ (9:1 hexanes:EtOAc); ${ }^{1} \mathrm{H}$ NMR $\left(300 \mathrm{MHz}, \mathrm{CDCl}_{3}\right) \delta 7.56-7.48(4 \mathrm{H}, \mathrm{m}), 7.38-7.31(1 \mathrm{H}, \mathrm{m}), 7.20(2 \mathrm{H}, \mathrm{t}, J=2.0 \mathrm{~Hz})$, $6.46(2 \mathrm{H}, \mathrm{t}, J=2.0 \mathrm{~Hz}) ;{ }^{13} \mathrm{C} \mathrm{NMR}\left(100 \mathrm{MHz}, \mathrm{CDCl}_{3}\right) \delta 140.89,129.69,125.75,120.66,119.46$, 110.55 .

4-Bromodiphenylamine (Barchiesi, E.; Bradamante, S.; Pagani, G. A. J. Chem. Soc. Perkin Trans. 2 1987, 1091-1096.)<smiles>Brc1ccc(Nc2ccccc2)cc1</smiles>

Reactions were performed at $40{ }^{\circ} \mathrm{C}$. Product was isolated as a clear, crystalline solid in $30 \%$ yield from $\mathrm{PhBF}_{3}{ }^{-} \mathrm{K}^{+}$and in $53 \%$ yield from $\mathrm{PhB}(\mathrm{OH})_{2}$ : $\mathrm{R}_{f}=0.40$ (3:1 hexanes:EtOAc); ${ }^{1} \mathrm{H}$ NMR $\left(300 \mathrm{MHz}, \mathrm{CDCl}_{3}\right) \delta 7.35$ - $7.22(4 \mathrm{H}, \mathrm{m}), 7.06-6.88(5 \mathrm{H}, \mathrm{m}), 5.63(1 \mathrm{H}, \mathrm{br} \mathrm{s}) ;{ }^{13} \mathrm{C} \mathrm{NMR}$ $\left(100 \mathrm{MHz}, \mathrm{CDCl}_{3}\right) \delta 142.56,142.53,132.31,129.90,121.79,119.17,118.43,112.74$.

\section{4-Anilinobenzoic acid methyl ester}<smiles>COC(=O)c1ccc(Nc2ccccc2)cc1</smiles>

Reactions were performed at room temperature. Product was isolated as a beige, crystalline solid in 35\% yield from $\mathrm{PhBF}_{3}{ }^{-} \mathrm{K}^{+}$and in $40 \%$ yield from $\mathrm{PhB}(\mathrm{OH})_{2}: \mathrm{R}_{f}=0.60$ (1:1 hexanes:EtOAc); ${ }^{1} \mathrm{H}$ NMR $\left(300 \mathrm{MHz}, \mathrm{CDCl}_{3}\right) \delta 7.92(2 \mathrm{H}, \mathrm{d}, J=8.5 \mathrm{~Hz}), 7.34(2 \mathrm{H}, \mathrm{t}, J=8.0 \mathrm{~Hz}), 7.17(2 \mathrm{H}, \mathrm{d}, J=$ $8.0 \mathrm{~Hz}), 7.07(1 \mathrm{H}, \mathrm{t}, J=8.0 \mathrm{~Hz}), 6.99(2 \mathrm{H}, \mathrm{d}, J=8.5 \mathrm{~Hz}), 6.07(1 \mathrm{H}, \mathrm{br} \mathrm{s}), 3.88(3 \mathrm{H}, \mathrm{s}) ;{ }^{13} \mathrm{C}$ NMR $\left(100 \mathrm{MHz}, \mathrm{CDCl}_{3}\right) \delta 167.16,148.27,141.03,131.65,129.67,123.28,121.26,120.62,114.75$, 51.89; IR (film) v 3343, 1695, 1591, 1521, 1496, 1434, 1328, 1282, 1172, 1109, 850, 769, 747, $693 \mathrm{~cm}^{-1}$; LRMS (EI): $\mathrm{m} / z$ (rel. intensity) = 228 (24), $227(100), 197(21), 196$ (95), $168(23)$, 167 (56), 166 (14), 98 (11), 84 (20), 77 (15), 51 (11); HRMS (EI): $\mathrm{m} / \mathrm{z}$ calcd. for $\left(\mathrm{M}^{+}\right)=$ 227.0946 , found $=227.0919$. 


\section{(2,6-Diethylphenyl)phenylamine}<smiles>CCc1cccc(CBr)c1Nc1ccccc1</smiles>

Reactions were performed at room temperature. Product was isolated as an amber oil in $34 \%$ yield from $\mathrm{PhBF}_{3}{ }^{-} \mathrm{K}^{+}$and in $49 \%$ yield from $\mathrm{PhB}(\mathrm{OH})_{2}: \mathrm{R}_{f}=0.70$ (3:1 hexanes:EtOAc); ${ }^{1} \mathrm{H}$ NMR $\left(300 \mathrm{MHz}, \mathrm{CDCl}_{3}\right) \delta 7.23-7.06(5 \mathrm{H}, \mathrm{m}), 6.71(1 \mathrm{H}, \mathrm{tt}, J=7.5,1.0 \mathrm{~Hz}), 6.47(2 \mathrm{H}, \mathrm{dd}, J=$ 8.5, $1.0 \mathrm{~Hz}), 5.13\left(1 \mathrm{H}\right.$, br s), $2.58(4 \mathrm{H}, \mathrm{q}, J=7.5 \mathrm{~Hz}), 1.14(6 \mathrm{H}, \mathrm{t}, J=7.5 \mathrm{~Hz}) ;{ }^{13} \mathrm{C}$ NMR $(100$

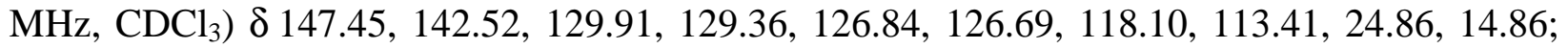
IR (film) v 3396, 3044, 2965, 2872, 1603, 1497, 1454, 1412, 1374, 1306, 1237, 1175, 1106, 1077, 1026, 994, 866, 798, 747, $693 \mathrm{~cm}^{-1}$; LRMS (EI): $\mathrm{m} / \mathrm{z}$ (rel. intensity) = 226 (29), 225 (100), 211 (24), 210 (92), 208 (20), 196 (18), 194 (14), 193 (13), 182 (15), 181 (32), 180 (53), 170 (15), 168 (11), 167 (13), 148 (12), 134 (13), 91 (12), 77 (19), 51 (12); HRMS (EI): m/z calcd. for $\left(\mathrm{M}^{+}\right)$ $=225.1518$, found $=225.1519$.

\section{(2-Methoxy-5-methylphenyl)phenylamine}<smiles>COc1ccc(C)cc1Nc1ccccc1</smiles>

$3 \mathbf{y}$

Reactions were performed at room temperature. Product was isolated as an orange oil in $51 \%$ yield from $\mathrm{PhBF}_{3}{ }^{-} \mathrm{K}^{+}$and in $66 \%$ yield from $\mathrm{PhB}(\mathrm{OH})_{2}: \mathrm{R}_{f}=0.60$ (3:1 hexanes:EtOAc); ${ }^{1} \mathrm{H}$ NMR $\left(300 \mathrm{MHz}, \mathrm{CDCl}_{3}\right) \delta 7.31(2 \mathrm{H}, \mathrm{t}, J=7.5 \mathrm{~Hz}), 7.17(3 \mathrm{H}, \mathrm{d}, J=7.5 \mathrm{~Hz}), 6.97(1 \mathrm{H}, \mathrm{tt}, J=$ $7.5,1.0 \mathrm{~Hz}), 6.80(1 \mathrm{H}, \mathrm{d}, J=8.0 \mathrm{~Hz}), 6.67(1 \mathrm{H}, \mathrm{dd}, J=8.0,1.0 \mathrm{~Hz}), 6.13(1 \mathrm{H}, \mathrm{br}), 3.88(3 \mathrm{H}$, s), $2.29(3 \mathrm{H}, \mathrm{s}) ;{ }^{13} \mathrm{C} \mathrm{NMR}\left(100 \mathrm{MHz}, \mathrm{CDCl}_{3}\right) \delta 146.45,142.97,132.90,130.41,129.42,121.23$, 120.26, 118.82, 115.64, 110.70, 55.94, 21.16; IR (film) v 3410, 3047, 2935, 2860, 2833, 1594, 1526, 1496, 1463, 1413, 1311, 1293, 1244, 1221, 1176, 1156, 1079, 1033, 956, 865, 795, 748, 725, 708, 694, $620 \mathrm{~cm}^{-1}$; LRMS (EI): $\mathrm{m} / z$ (rel. intensity) = 214 (19), 213 (100), 199 (15), 198 (89), 197 (17), 183 (33), 170 (26), 155 (11), 154 (11), 77 (12); HRMS (EI): $\mathrm{m} / z$ calcd. for $\left(\mathrm{M}^{+}\right)=$ 213.1154 , found $=213.1160$. 


\section{Products of Experiments Summarized in Table 3}

8-(4-Acetylphenyl)-1,4-dioxa-8-azaspiro[4.5]decane (Ogawa, K.; Kaji, M.; Kagawa, H.; Sagawa, M.; Kakuta, A. Acta Crystallogr. Sect. C: Cryst. Struct. Commun. 1994, 50, $95-$ 97.)

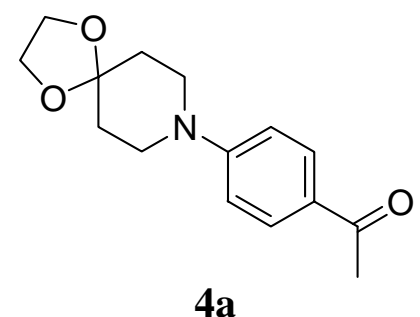

Reaction was performed at $40{ }^{\circ} \mathrm{C}$. Product was isolated as a clear, crystalline solid in $93 \%$ yield from $\mathrm{PhB}(\mathrm{OH})_{2}: \mathrm{R}_{f}=0.10$ (3:1 hexanes:EtOAc); ${ }^{1} \mathrm{H} \mathrm{NMR}\left(300 \mathrm{MHz}, \mathrm{CDCl}_{3}\right) \delta 7.84(2 \mathrm{H}$, ddd, $J$ $=10.0,5.0,3.0 \mathrm{~Hz}), 6.85(2 \mathrm{H}$, ddd, $J=10.0,5.0,3.0 \mathrm{~Hz}), 3.98(4 \mathrm{H}, \mathrm{s}), 3.50(4 \mathrm{H}, \mathrm{dd}, J=6.0,6.0$ $\mathrm{Hz}), 2.50(3 \mathrm{H}, \mathrm{s}), 1.78(4 \mathrm{H}, \mathrm{dd}, J=6.0,6.0 \mathrm{~Hz}) ;{ }^{13} \mathrm{C} \mathrm{NMR}\left(100 \mathrm{MHz}, \mathrm{CDCl}_{3}\right) \delta 196.54,153.58$, $130.63,127.21,113.60,107.14,64.55,45.95,34.38,26.22$.

\section{8-(3-Chlorophenyl)-1,4-dioxa-8-azaspiro[4.5]decane}

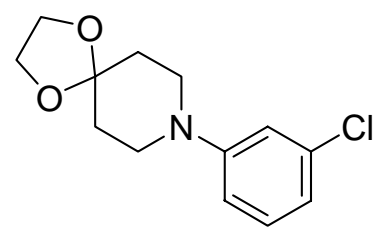

4b

Reaction was performed at $40{ }^{\circ} \mathrm{C}$. Product was isolated as a clear oil in $85 \%$ yield from $\mathrm{PhB}(\mathrm{OH})_{2}: \mathrm{R}_{f}=0.30$ (3:1 hexanes:EtOAc); ${ }^{1} \mathrm{H}$ NMR $\left(300 \mathrm{MHz}, \mathrm{CDCl}_{3}\right) \delta 7.14(1 \mathrm{H}, \mathrm{t}, J=8.0$ $\mathrm{Hz}), 6.89(1 \mathrm{H}, \mathrm{t}, J=2.0 \mathrm{~Hz}), 6.82-6.75(2 \mathrm{H}, \mathrm{m}), 3.98(4 \mathrm{H}, \mathrm{s}), 3.33(4 \mathrm{H}, \mathrm{dd}, J=6.0,6.0 \mathrm{~Hz})$, $1.81(4 \mathrm{H}, \mathrm{dd}, J=6.0,6.0 \mathrm{~Hz}) ;{ }^{13} \mathrm{C}$ NMR $\left(100 \mathrm{MHz}, \mathrm{CDCl}_{3}\right) \delta 152.00,135.03,130.18,119.01$, 116.27, 114.42, 107.15, 64.50, 47.35, 34.47; IR (film) v 2958, 2883, 1595, 1563, 1487, 1466, 1365, 1231, 1144, 1097, 1037, 945, 768, $683 \mathrm{~cm}^{-1}$; LRMS (EI): $\mathrm{m} / z$ (rel. intensity) $=255(37)$, 254 (21), 253 (100), 210 (10), 208 (30), 194 (11), 192 (21), 168 (16), 167 (18), 166 (42), 141 (26), 140 (15), 139 (74), 138 (20), 111 (13), 86 (11); HRMS (EI): $\mathrm{m} / z$ calcd. for $\left(\mathrm{M}^{+}\right)=$ 253.0869 , found $=253.0870$. 


\section{8-(3-Nitrophenyl)-1,4-dioxa-8-azaspiro[4.5]decane}

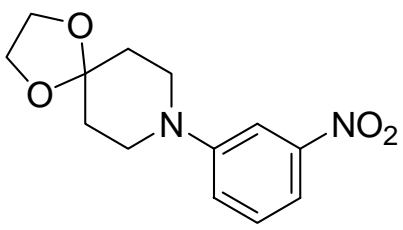

$4 c$

Reaction was performed at $40{ }^{\circ} \mathrm{C}$. Product was isolated as a bright yellow oil in $84 \%$ yield from $\mathrm{PhB}(\mathrm{OH})_{2}: \mathrm{R}_{f}=0.25$ (3:1 hexanes:EtOAc); ${ }^{1} \mathrm{H}$ NMR $\left(300 \mathrm{MHz}, \mathrm{CDCl}_{3}\right) \delta 7.68(1 \mathrm{H}, \mathrm{t}, J=2.0$ $\mathrm{Hz}), 7.57(1 \mathrm{H}, \mathrm{ddd}, J=8.0,2.0,1.0 \mathrm{~Hz}), 7.32(1 \mathrm{H}, \mathrm{t}, J=8.0 \mathrm{~Hz}), 7.16(1 \mathrm{H}, \mathrm{ddd}, J=8.0,2.0,1.0$ $\mathrm{Hz}), 3.97(4 \mathrm{H}, \mathrm{s}), 3.40(4 \mathrm{H}, \mathrm{dd}, J=6.0,6.0 \mathrm{~Hz}), 1.78(4 \mathrm{H}, \mathrm{dd}, J=6.0,6.0 \mathrm{~Hz}) ;{ }^{13} \mathrm{C}$ NMR $(100$ $\left.\mathrm{MHz}_{,} \mathrm{CDCl}_{3}\right) \delta 151.34,149.35,129.79,121.52,113.27,109.96,106.86,64.51,46.98,34.27$; IR (film) v 2960, 2886, 1616, 1526, 1349, 1234, 1144, 1112, 1036, 946, 856, 737, $674 \mathrm{~cm}^{-1}$; LRMS (EI): $m / z$ (rel. intensity) = 265 (19), 264 (93), 219 (46), 204 (12), 203 (35), 178 (24), 177 (48), 151 (13), 150 (100), 149 (10), 132 (12), 105 (15), 104 (15), 99 (17), 86 (14), 77 (15); HRMS (EI): $m / z$ calcd. for $\left(\mathrm{M}^{+}\right)=264.1110$, found $=264.1109$.

\section{8-(4-Methoxyphenyl)-1,4-dioxa-8-azaspiro[4.5]decane}

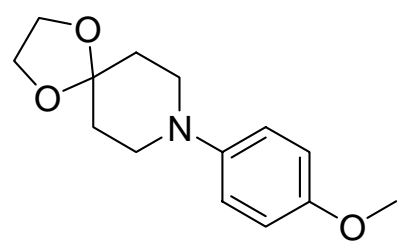

4d

Reaction was performed at $40{ }^{\circ} \mathrm{C}$. Product was isolated as a clear, crystalline solid in $95 \%$ yield from $\mathrm{PhB}(\mathrm{OH})_{2}: \mathrm{mp}=60-61{ }^{\circ} \mathrm{C}$ (hexanes); $\mathrm{R}_{f}=0.20$ (3:1 hexanes:EtOAc); ${ }^{1} \mathrm{H}$ NMR (300 $\left.\mathrm{MHz} \mathrm{CDCl}_{3}\right) \delta 6.92(2 \mathrm{H}, \mathrm{ddd}, J=10.0,6.0,3.5 \mathrm{~Hz}), 6.82(2 \mathrm{H}, \mathrm{ddd}, J=10.0,6.0,3.5 \mathrm{~Hz}), 3.98$ $(4 \mathrm{H}, \mathrm{s}), 3.76(3 \mathrm{H}, \mathrm{s}), 3.18(4 \mathrm{H}, \mathrm{dd}, J=5.5,5.5 \mathrm{~Hz}), 1.86(4 \mathrm{H}, \mathrm{dd}, J=5.5,5.5 \mathrm{~Hz}) ;{ }^{13} \mathrm{C} \mathrm{NMR}$ $\left(100 \mathrm{MHz}, \mathrm{CDCl}_{3}\right) \delta 153.82,145.74,119.05,114.44,107.16,64.41,55.62,49.47,34.95$; IR (film) v 2956, 2821, 1512, 1466, 1442, 1417, 1368, 1288, 1249, 1224, 1180, 1115, 1036, 949, 896, 832, $704 \mathrm{~cm}^{-1}$; LRMS (EI): $\mathrm{m} / \mathrm{z}$ (rel. intensity) = 250 (26), 249 (100), 235 (11), 234 (51), 204 (12), 188 (10), 163 (13), 162 (42), 136 (12), 135 (63), 133 (18), 121 (11), 120 (41), 99 (11), 92 (10), 86 (11), 84 (16), 55 (12), 51 (12); HRMS (EI): $\mathrm{m} / z$ calcd. for $\left(\mathrm{M}^{+}\right)=249.1365$, found = 249.1361 . 


\section{8-(Pyridin-3-yl)-1,4-dioxa-8-azaspiro[4.5]decane}

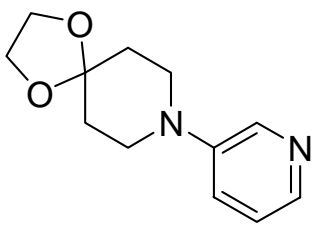

$4 e$

Reaction was performed at $40{ }^{\circ} \mathrm{C}$. Product was isolated as light orange oil in $55 \%$ yield from $\mathrm{PhB}(\mathrm{OH})_{2}: \mathrm{R}_{f}=0.10$ (1:1 CH2Cl2:EtOAc); ${ }^{1} \mathrm{H}$ NMR (300 MHz, $\left.\mathrm{CDCl}_{3}\right) \delta 8.31(1 \mathrm{H}, \mathrm{s}), 8.05$ $(1 \mathrm{H}, \mathrm{d}, J=3.5 \mathrm{~Hz}), 7.18(1 \mathrm{H}, \mathrm{ddd}, J=8.5,3.5,1.5 \mathrm{~Hz}), 7.11(1 \mathrm{H}, \mathrm{dd}, J=8.5,3.5 \mathrm{~Hz}), 3.98(4 \mathrm{H}$, s), $3.34(4 \mathrm{H}, \mathrm{dd}, J=5.5,5.5 \mathrm{~Hz}), 1.82(4 \mathrm{H}, \mathrm{dd}, J=5.5,5.5 \mathrm{~Hz}) ;{ }^{13} \mathrm{C} \mathrm{NMR}\left(100 \mathrm{MHz}, \mathrm{CDCl}_{3}\right)$ $\delta 146.77,140.50,139.20,123.60,122.90,106.98,64.53,47.24,34.46$; IR (film) v 2958, 2884, $1672,1582,1488,1426,1365,1235,1144,1102,1052,1035,946,930,799,708,670,614 \mathrm{~cm}^{-1}$; LRMS (EI): $m / z$ (rel. intensity) = 221 (14), 220 (82), 175 (32), 159 (26), 134 (17), 133 (55), 107 (15), 106 (100), 105 (29), 99 (20), 86 (20), 78 (21), 55 (11), 51 (10); HRMS (EI): m/z calcd. for $\left(\mathrm{M}^{+}\right)=220.1212$, found $=220.1217$.

\section{8-o-Tolyl-1,4-dioxa-8-azaspiro[4.5]decane}

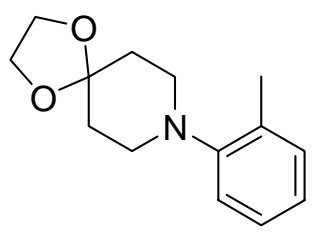

4f

Reaction was performed at $40{ }^{\circ} \mathrm{C}$. Product was isolated as a pale yellow oil in $61 \%$ yield from $\mathrm{PhB}(\mathrm{OH})_{2}: \mathrm{R}_{f}=0.33$ (9:1 hexanes:EtOAc); ${ }^{1} \mathrm{H} \mathrm{NMR}\left(300 \mathrm{MHz}, \mathrm{CDCl}_{3}\right) \delta 7.24-7.18(2 \mathrm{H}, \mathrm{m})$, $7.07(1 \mathrm{H}, \mathrm{dd}, J=7.5,1.0 \mathrm{~Hz}), 6.99(1 \mathrm{H}, \mathrm{dt}, J=7.5,1.0 \mathrm{~Hz}), 4.02(4 \mathrm{H}, \mathrm{s}), 3.01(4 \mathrm{H}, \mathrm{dd}, J=5.5$, $5.5 \mathrm{~Hz}), 2.34(3 \mathrm{H}, \mathrm{s}), 1.91(4 \mathrm{H}, \mathrm{dd}, J=5.5,5.5 \mathrm{~Hz}) ;{ }^{13} \mathrm{C} \mathrm{NMR}\left(100 \mathrm{MHz}, \mathrm{CDCl}_{3}\right) \delta 151.95$, 132.76, 131.05, 126.60, 123.14, 119.38, 107.30, 64.41, 50.27, 35.79, 17.90; IR (film) v 2955, 2880, 2823, 1599, 1493, 1470, 1363, 1328, 1217, 1142, 1101, 1038, 931, 899, 762, 724, $666 \mathrm{~cm}^{-}$ 1; LRMS (EI): $m / z$ (rel. intensity) = 234 (22), 233 (100), 232 (16), 188 (41), 172 (19), 147 (15), 146 (55), 132 (17), 119 (42), 118 (44), 91 (18); HRMS (EI): $m / z$ calcd. for $\left(\mathrm{M}^{+}\right)=233.1416$, found $=233.1411$. 
STANDARD 1H OBSERVVE

Pu1se Sequence: s2pul

solvent: $\operatorname{CDC13}$

Temo. $25.0 \mathrm{C} / 298.1 \mathrm{~K}$

Marcury-300

$3 g$

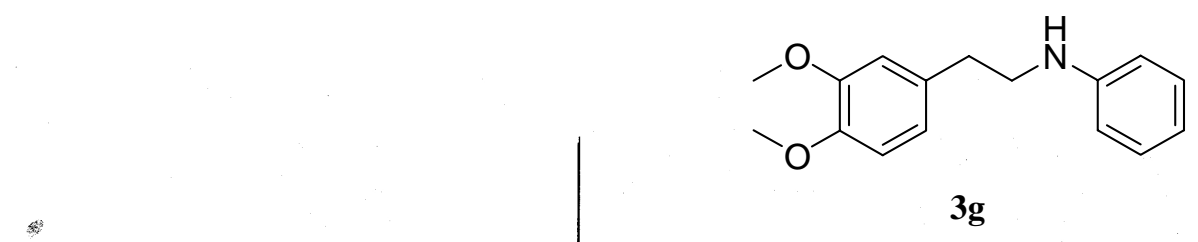

Pulse 79.0 degrees

Acq. time 2.276 wec

width $4492.4 \mathrm{~Hz}$

16 repetitions

DATA PROCESSTMO

Line broadening $0.2 \mathrm{~Hz}$

FT aize 65536

Total timo 0 min, 12 sec

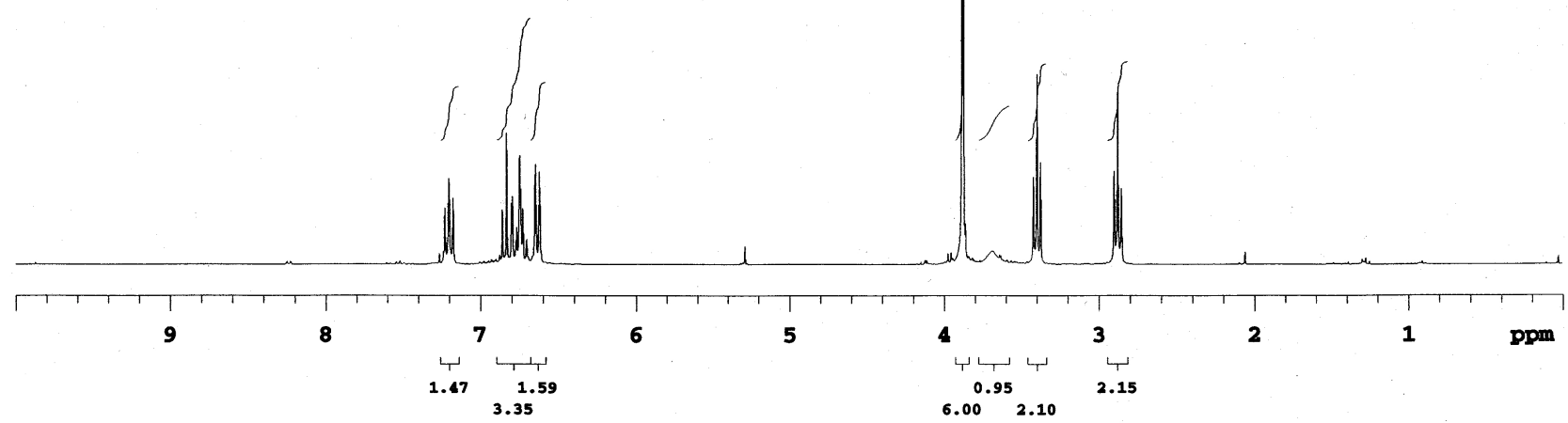

13C OBSERVE

Pulse Sequence: spul

Solvent: $\operatorname{CDC13}$

Tem. 25.0 C / $298.1 \mathrm{~K}$

Morcury-300 "morcury300

Pulse 48.6 dogrees

Acd. time 1.000

width $18797.0 \mathrm{Bz}$

368 repetitions

OBSERVE C13, 75.4478965 MAE

DECOUPLE H1, 300.0525807 MFE

Power 40 de

continuously on

warrz-16 modulated

DATA PROCESSTrFa

IIno broadening $1.0 \mathrm{~Hz}$

FT size 131072

Total timo $1 \mathrm{hr}, 2 \mathrm{~min}, 5 \mathrm{sec}$

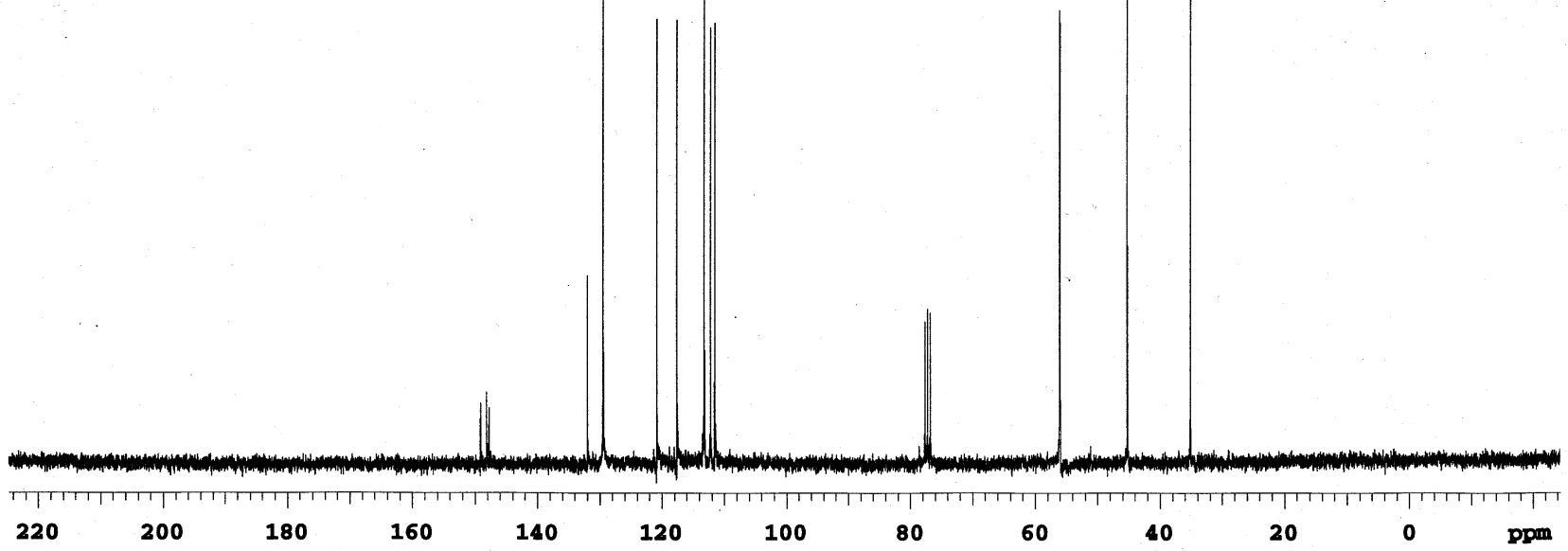


Pulse sequence: s2pul

Solvent: CDC13

Ambient temperature

GarrMI-300BB "geminis 300"<smiles>COCCNc1ccccc1</smiles>

Relax. delay $1.000 \mathrm{sec}$

pulse 45.0 degrees.

Acq. time $1.998 \mathrm{sec}$

W1dth 4500.5 Hz

16 repetitions

OBSERVE H1, 300.0738366 YRz

DATA PROCESSTMO

Line broadening $0.2 \mathrm{~Hz}$

Fine broadon: 32768

Total time 0 min, $53 \mathrm{sec}$

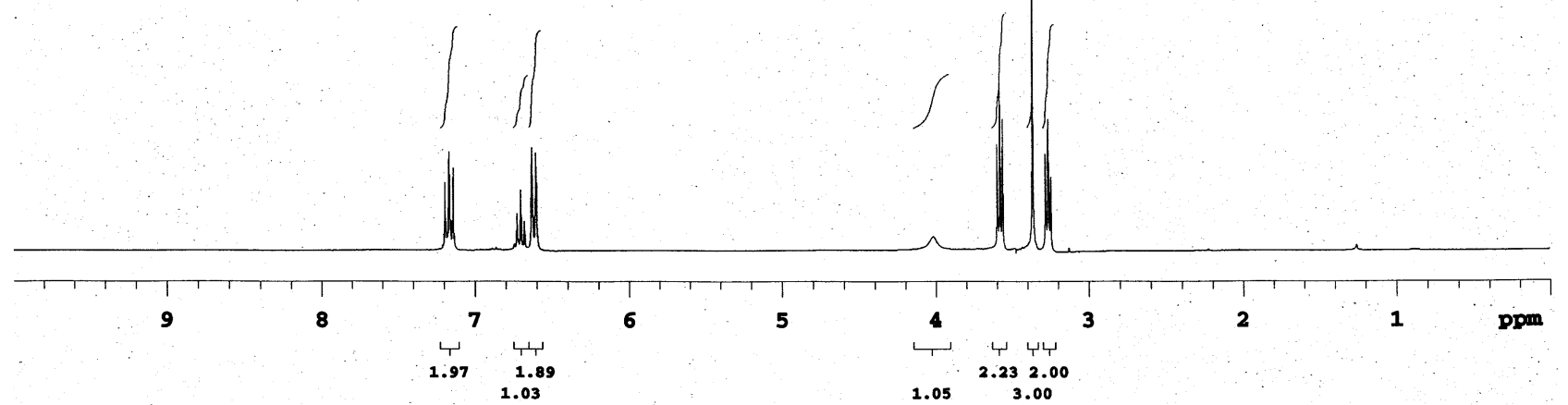

13C OBSERVE

Pulse Sequence: spul

Solvent: CDC13

Ambient temperature

Ambiont tomperature

Pulse 28.6 degrees

Acq. time $1.815 \mathrm{sec}$

width $18761.7 \mathrm{~Hz}$

136 repotition

OBSERVI C13, 75.4536182 uHz

DECOUPIS H1, $300.0753189 \mathrm{kHz}$

Powor 33 dB

minzz-16 modulat

DATA PROCESSTMC

Line broadening 1.0 HE

Fr size 131072

Total timo $10 \mathrm{~min}, 36 \mathrm{sec}$

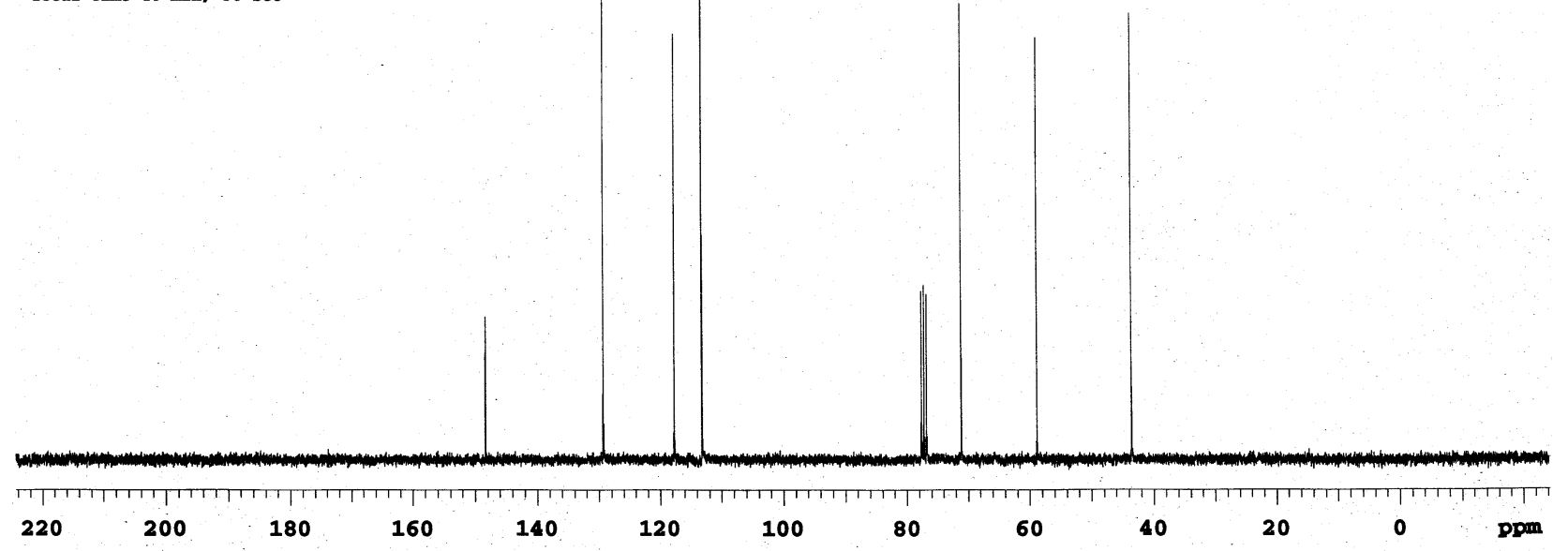


STAMBARD 1H OBgERVE

Pulse sequence: E2pul

solvent: CDC13

Tamp. $25.0 \mathrm{C} / 298.1 \mathrm{~K}$

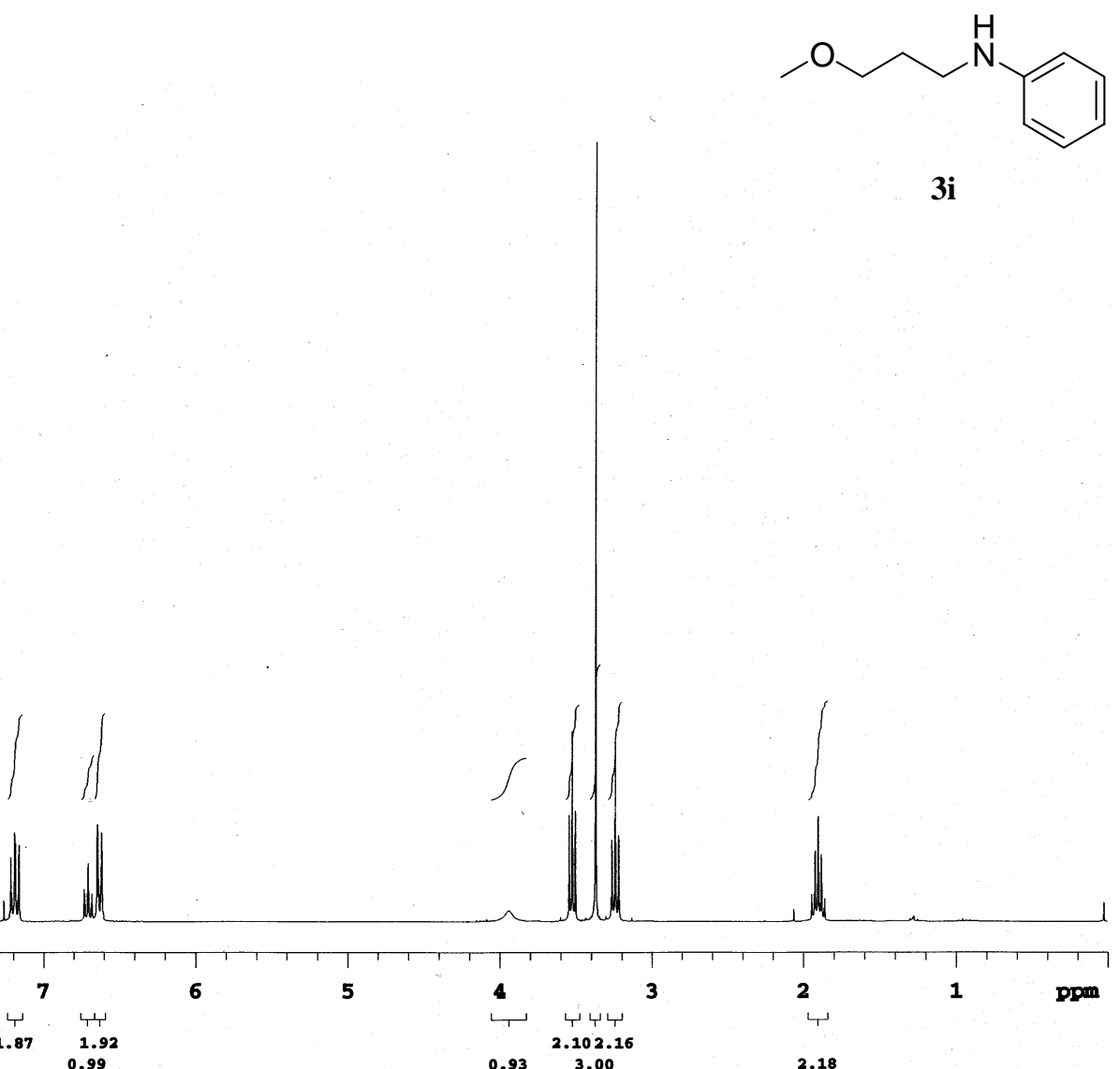

morcury-300 morcury300"

3i

Relax. delay $5.000 \mathrm{sec}$

Pulse 79.0 degreess

cq. 2.276 sec

wath 1492.4 HE

16 repetitions

OBsiervE H1, 300

DATA PROCEssing $0.2 \mathrm{~Hz}$

L1ne broaden:

Total timo 2 min, 2 sec

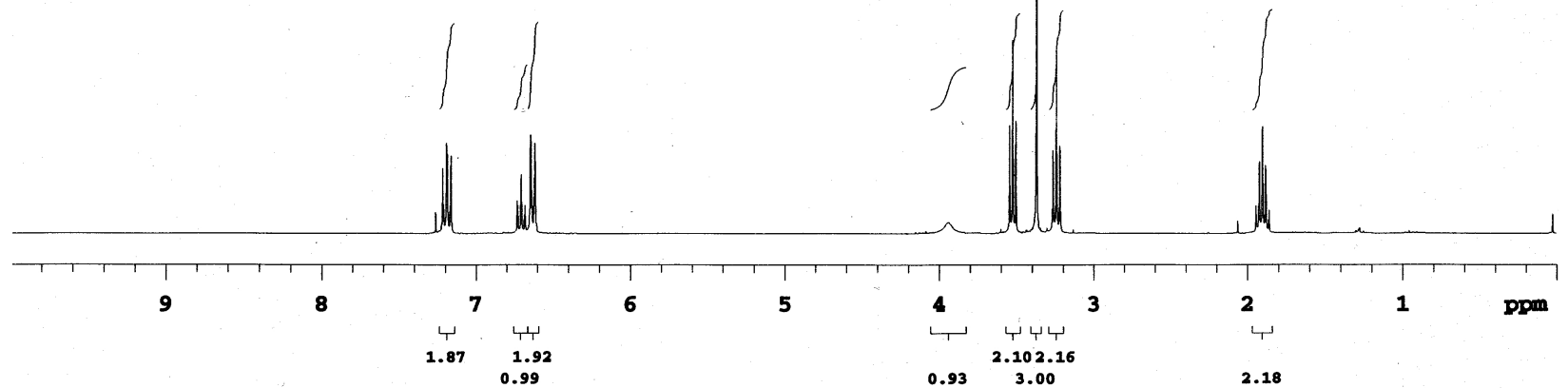

13C OBgarve

Pulse sequence: 2pul

Solvent: $\mathrm{CDC}_{3} 3$

Temo. 25.0 C / $298.1 \mathrm{~K}$

Morcury-300 "morcury 300 "

Pulse 48.6 degrees

Acq. time $1.000=0$

width 18797.0 B

224 ropetitions

OBSERVE C13, 75.4478928 $\mathrm{MHZ}$

Power 40 dB

continuously on

MaLrz-16 modulated

DATA PROCESSIDM

IIne broadening $1.0 \mathrm{H}$

FT s1ze 131072

Total time 1 hx, 2 min, 5 soc

$0.93 \quad 3.00$

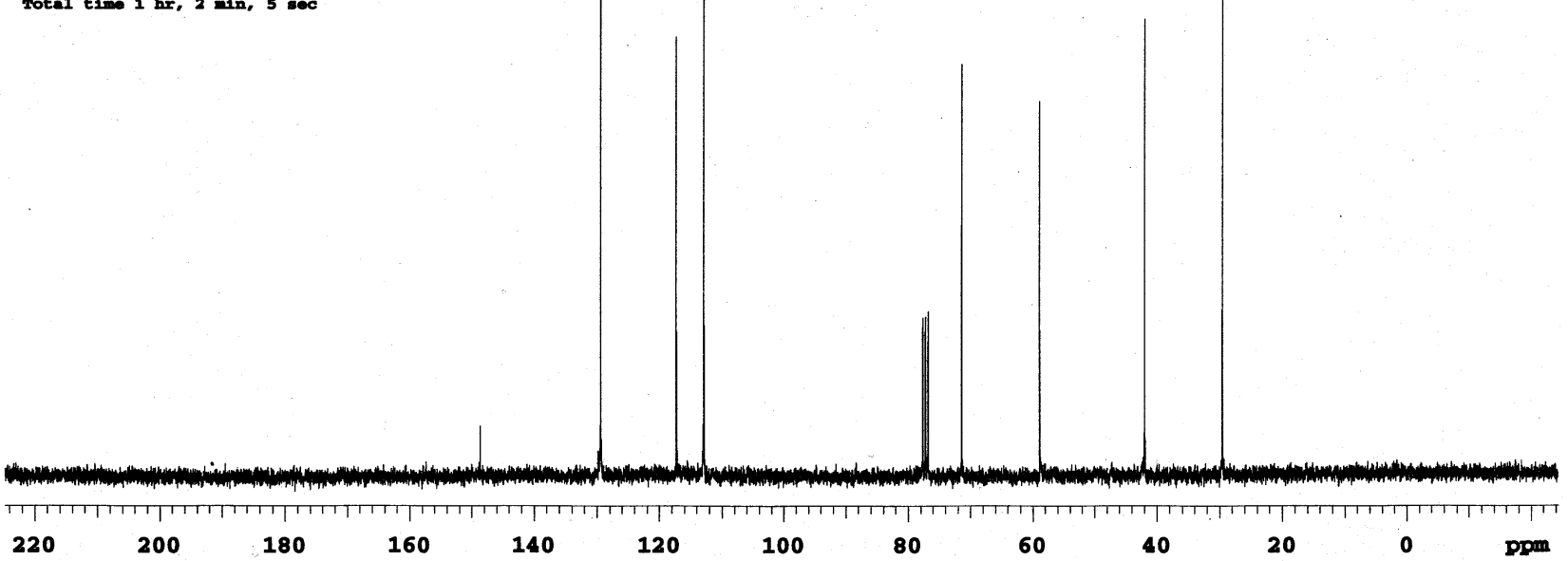




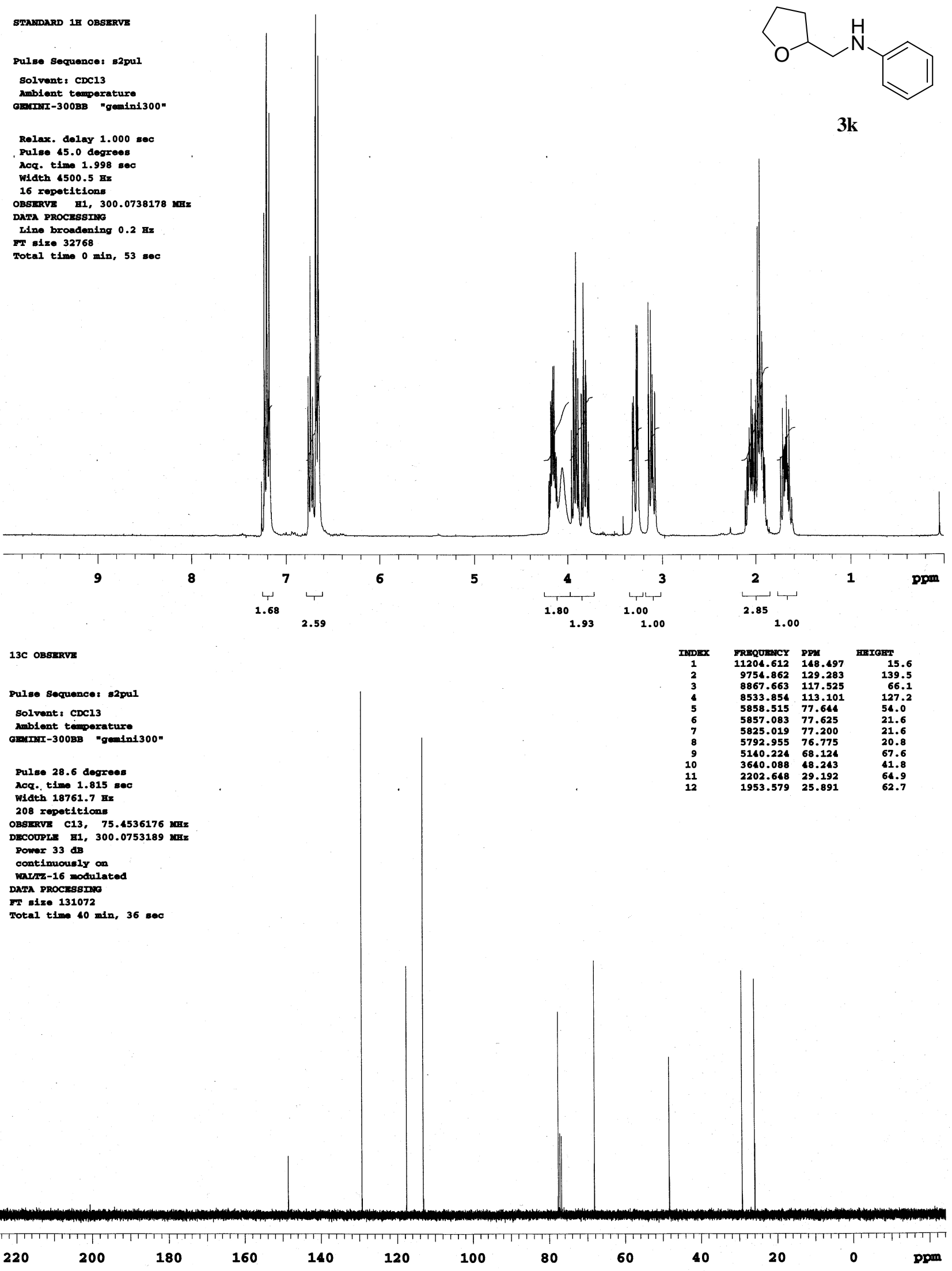


STMADARD IH OBgIERVE

Puleo sequences spul

Solvont: $\mathrm{CDC}_{13}$

Ambiont temperature

GenT-300BB "geminin $1300=$

Rolax. delay $1.000 \mathrm{sec}$

Pulse 45.0 degrees

Acq. tim $1.998 \mathrm{sec}$

Width $4500.5 \mathrm{HI}$

16 ropotitions

DAYA PROCEsSTro

Line broadening 0.2 Ex

FT size 32768

Total timo 0 min, $53 \mathrm{sec}$

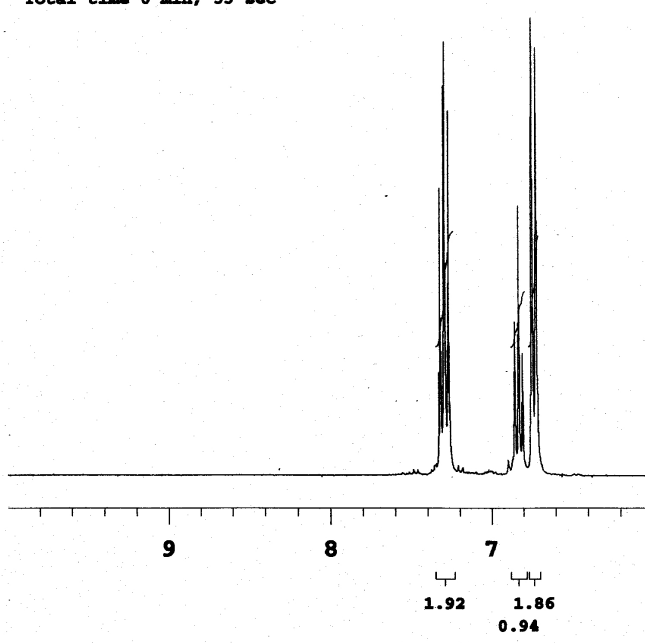

31

13C OBsarvis

Pulse sequence: s2pul

solvent: CDC13

Ambient tomperature

Gerrar-300Bs "gemini300"

Pulee 28.6 degrees

ncq. tim 1.815

Width $18761.7 \mathrm{Hx}$

96 ropetitions 75.4536162 war

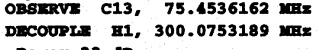

Power 33 dB

continuous $1 y$ on

mul/1z-16 modulat

DATM PROCESBINO

Line broadening $1.0 \mathrm{~Hz}$

FT size 131072

Total time $40 \mathrm{~min}, 36 \mathrm{sec}$

6

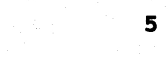

5

0.94

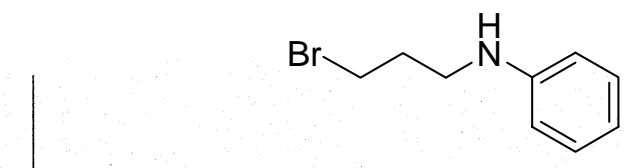

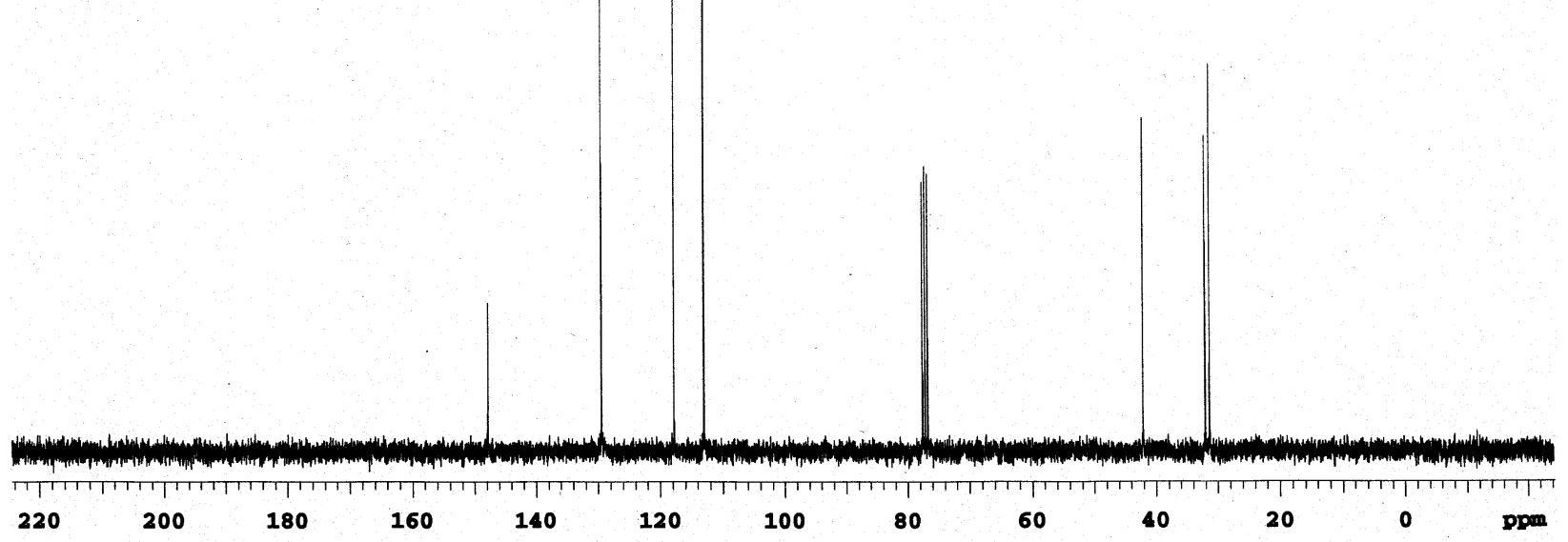


STMADARD 1H OBSERVE

Pulse Sequence: spoul

Solvent: CDC13

GerTsI-300BB "gemini300"

Rolax. dolay $1.000 \mathrm{sec}$

Pulse 45.0 degrees

width $4500.5 \mathrm{~Hz}$

16 repetition:

OBSERVI H1, 300.0737984

DATA PROCEsstme

Line brodening $0.2 \mathrm{Hs}$

FT size 32768

Total time 0 min, $53 \mathrm{sec}$

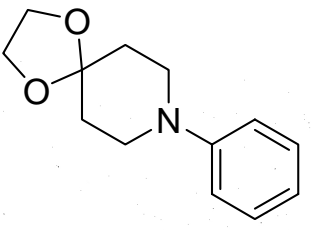

$3 q$

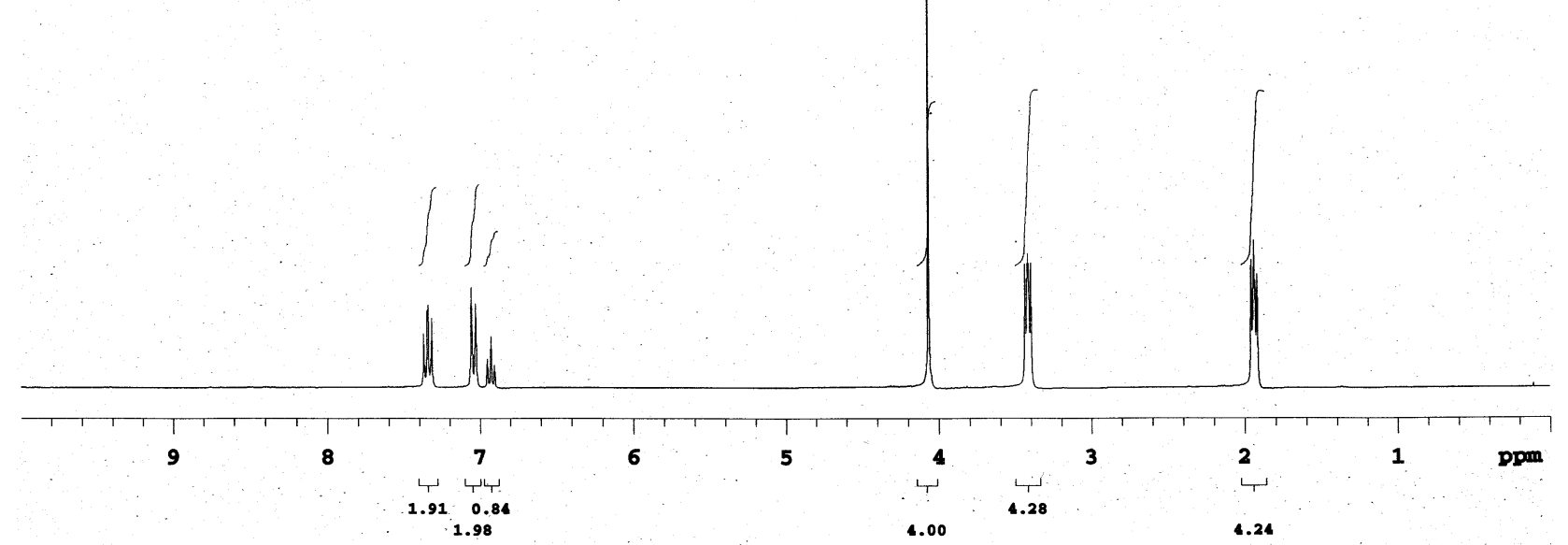

13C OBgERVE

Pulse sequence: s2pul

Solvent: $\mathrm{CDCl}_{3}$

Ambient temoerature

Georrr-300BB "gemini300"

Pulse 28.6 dogree

Acq. time 1.815

Width $18761.7 \mathrm{H}$

32 repatitions

OBSIARYE C13, 75.4536162 are

DECOUPLI: E1, 300.0753189

Power 33 de

continuously on

wnI/1z-16 modulated

DATA PROCRSSTMC

Ilno broadening $1.0 \mathrm{~F}$

FT size 131072

Total timo 49 min, 41
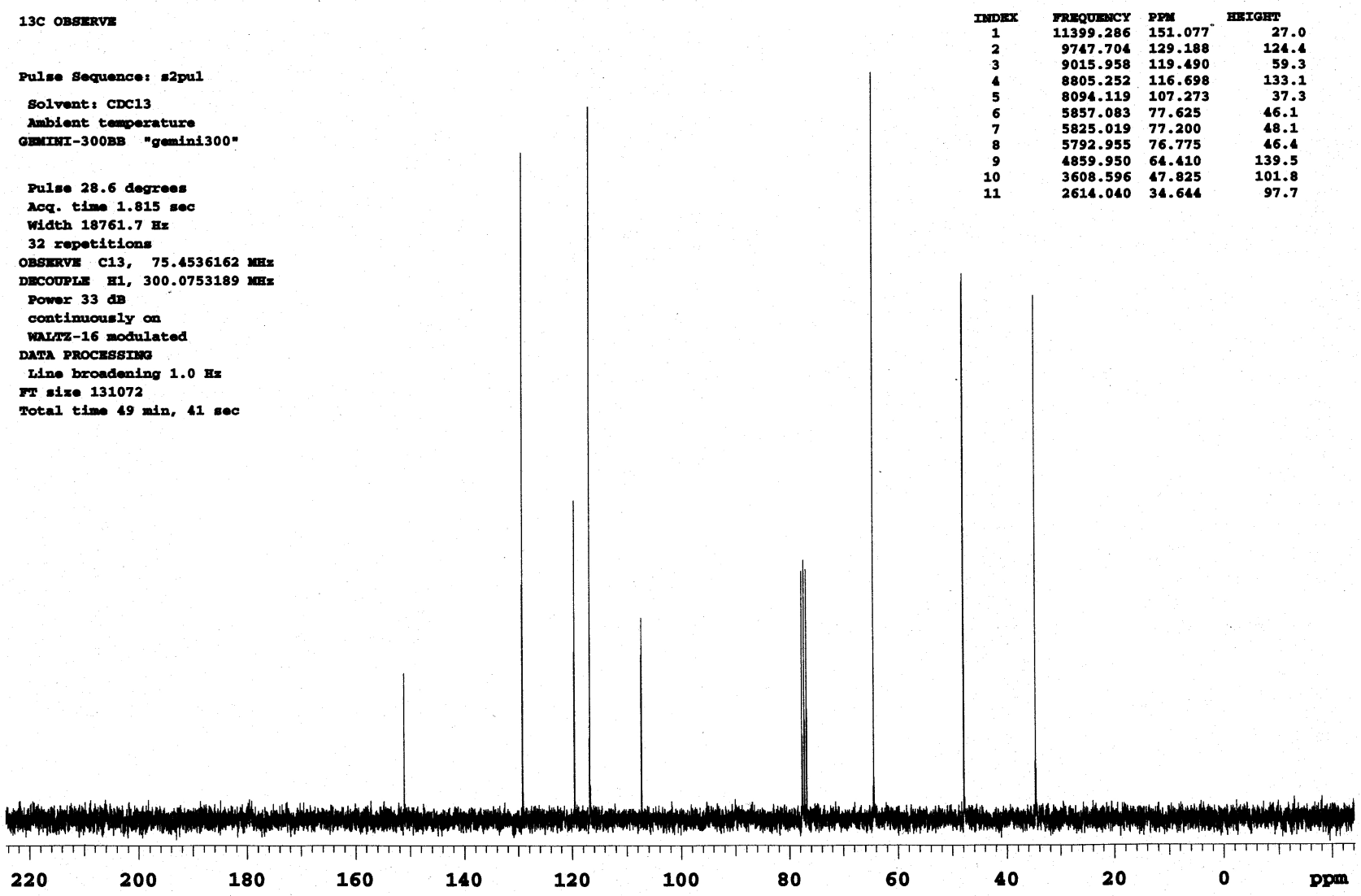
STANDARD 1H OBgERVE

Pulse Sequences s2pul

Solvent: CDC13

Temp. $25.0 \mathrm{C} / 298.1 \mathrm{~K}$

Morcury-300 "morcury 300 "

Pulse 79.0 degrees

Acq. time 2.276

width $4492.4 \mathrm{~Hz}$

16 repotition:

OBSERVE H1, $300.0510808 \mathrm{MHZ}$

DATA PROCESSIMG

Line broadening $0.2 \mathrm{~Hz}$

FT sixe 65536

Total time 0 min, $12 \mathrm{sec}$<smiles>COC(=O)c1ccc(Nc2ccccc2)cc1</smiles>

3w

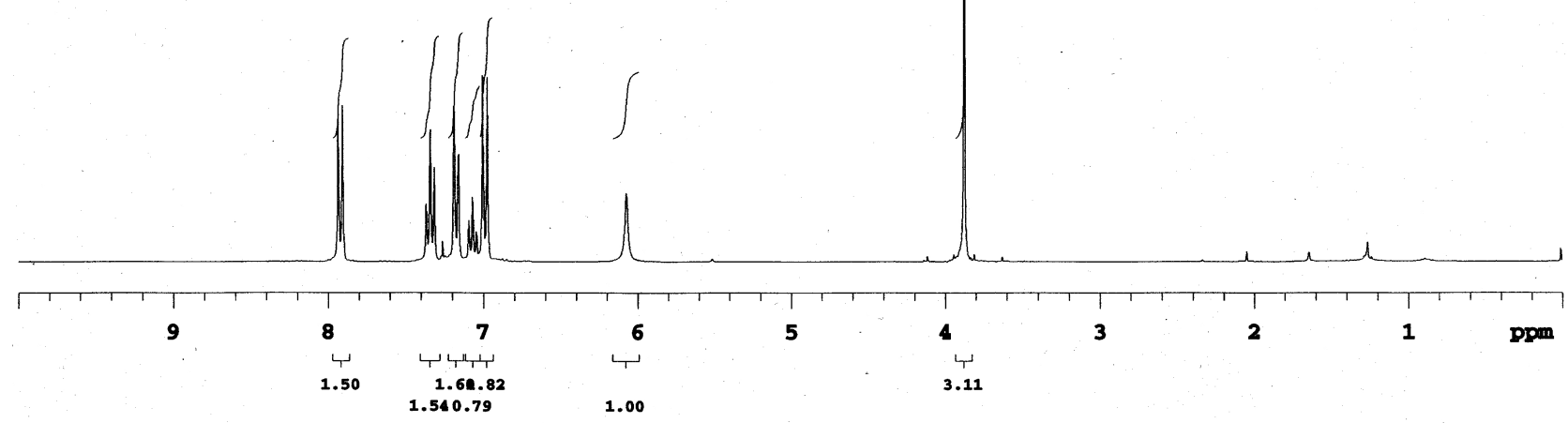

13C OBgERVE

Pulse Sequence: spul

Solvent: $\mathrm{CDCl}_{3}$

Temp. 25.0 C / $298.1 \mathrm{x}$

Wercury-300 =morcury 300

Pulse 48.6 degrees

leq. time 1.000

Width 18797.0 H:

88 repetition

OBSERVI C13, 75.4178910 was

DECOUPLE H1, 300.0525807 wat

Power 40 ab

continuously on

nulsz-16 modulated

DATA PROCESBITNO

Iine broadening $1.0 \mathrm{z}$

FT =1ze 131072

Total tim $1 \mathrm{hr}, 2$ min, 5 sec

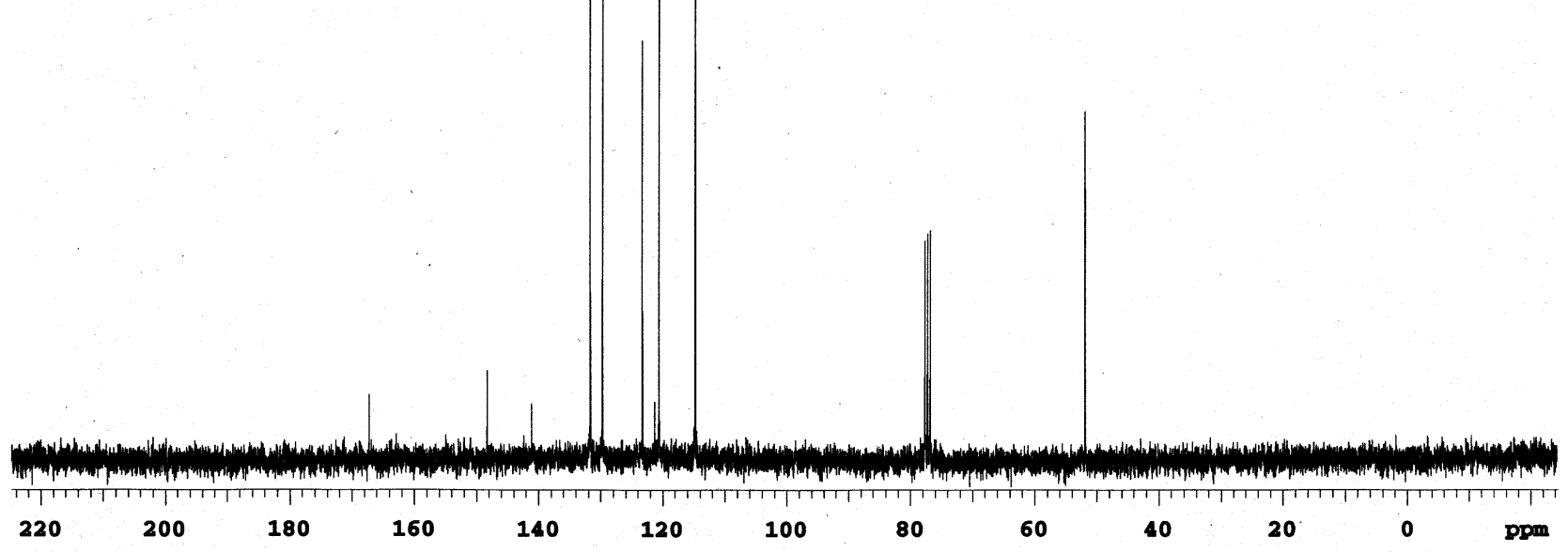



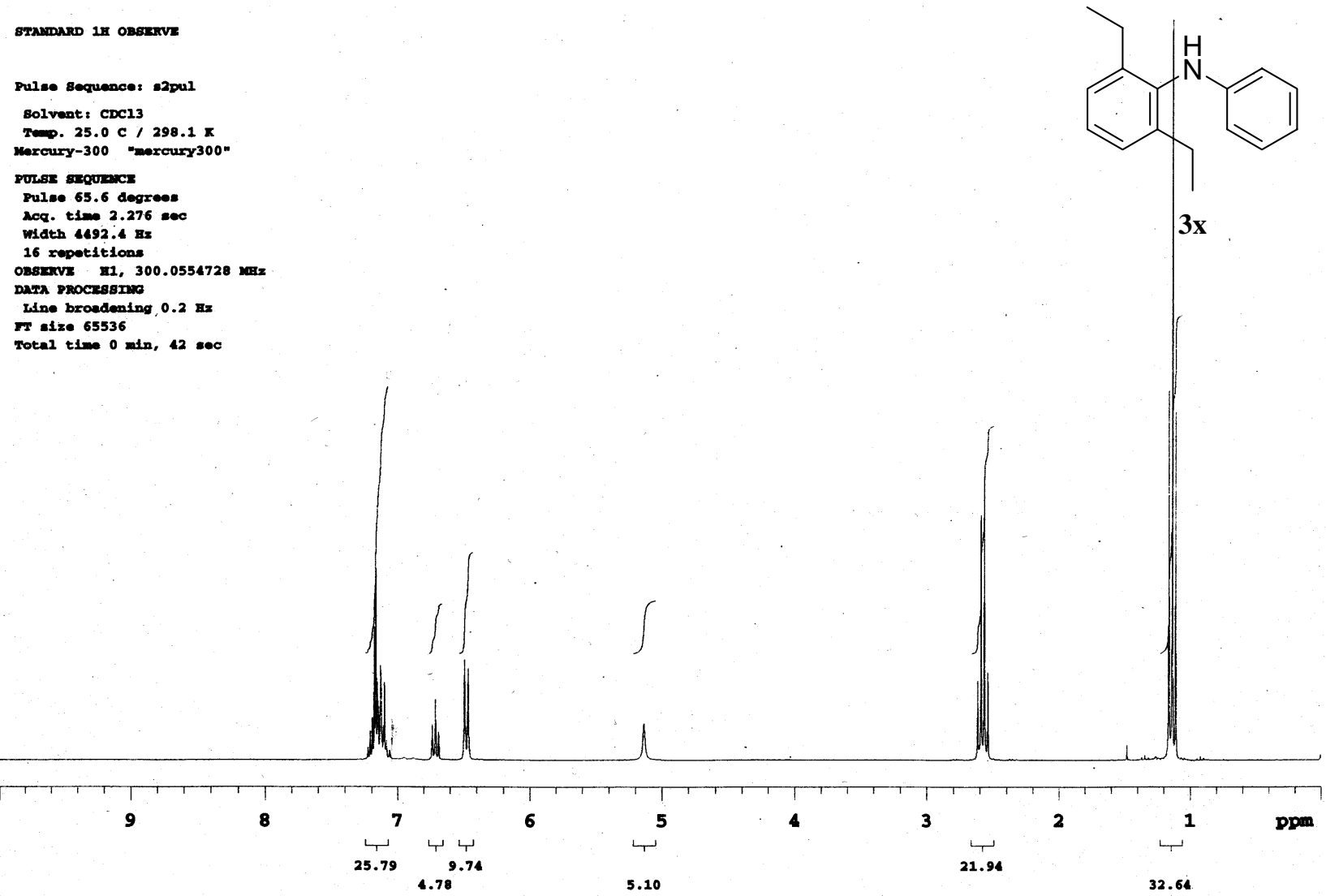

13c Osgaxye
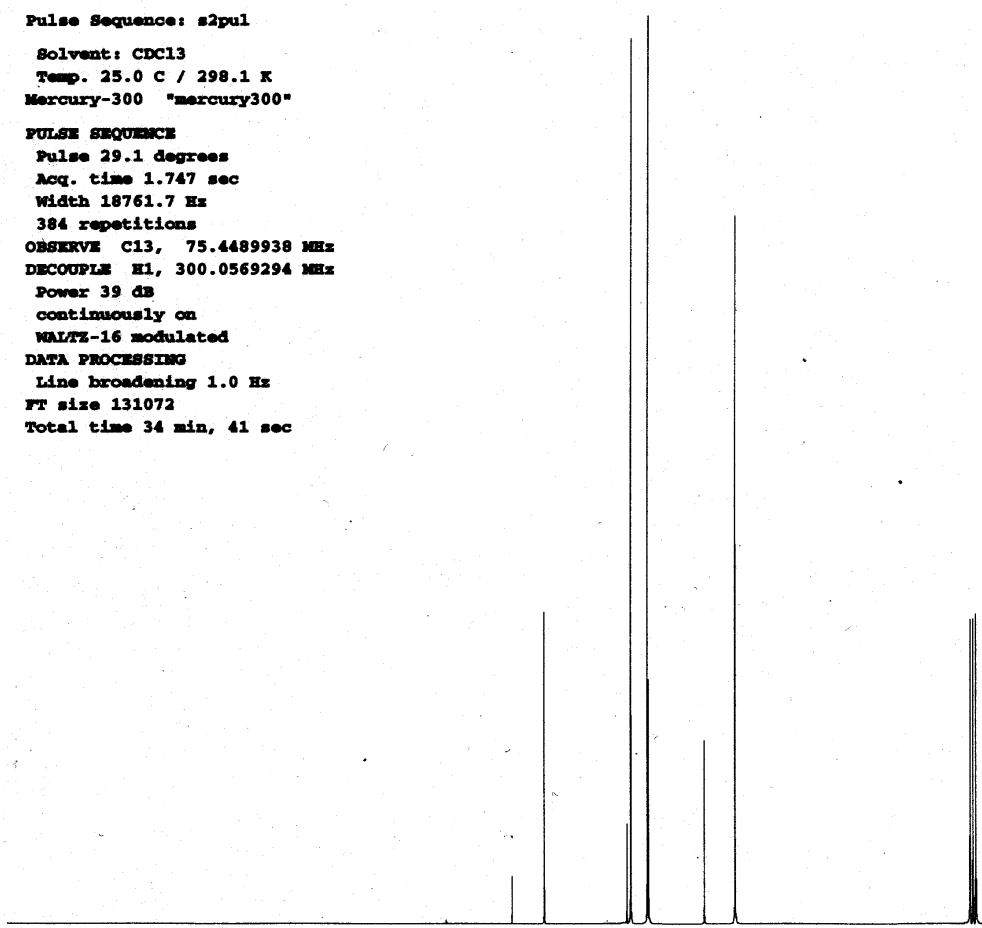

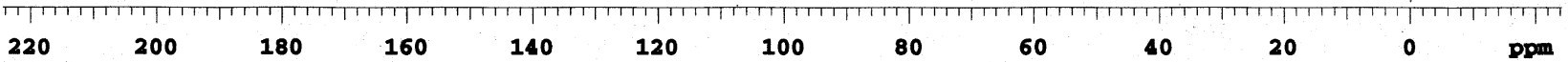


STAMDARD 1I OBSERVE

Pulse sequence: 2 pul

Solvent: $\mathrm{CDC13}$

Teap. $25.0 \mathrm{C} / 298.1 \mathrm{k}$

(xyry 300

79.0 degrees

Acq. tim 2.276

width $492.4 \mathrm{~Hz}$

16 ropetitions

DATA PROCESSIMG

Line broadoning $0.2 \mathrm{Hx}$

FT size 65536

rotal timo 0 min, 12 sec<smiles>COc1ccc(C)cc1Nc1ccccc1</smiles>

$3 y$

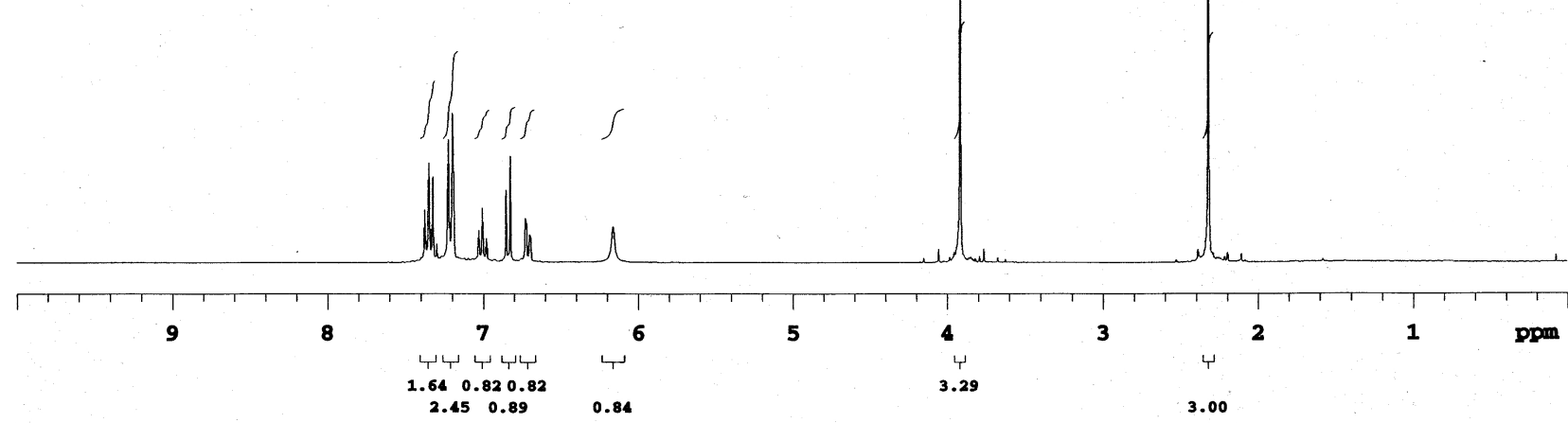

13C OBgEerVE

Pulse sequence: s2pul

Solvent: $\mathrm{CDCl}_{3}$

Temp. 25.0 C / $298.1 \mathrm{~K}$

Morcury-300 marcury300

Pulse 48.6 degrees

Acq. time 1.000 sec

width $18797.0 \mathrm{~m}$

296 ropotitions

OBSERVE C13, 75.4478925 MII:

DECOUPHE H1, 300.0525807 atiz

Power 10 dB

continuousiy on

marnz-16 modulated

DAMA PROCESBIMG

Line broadening $1.0 \mathrm{~B}$

FT size 131072

Total time $1 \mathrm{hx}, 2 \mathrm{~min}, 5 \mathrm{sec}$

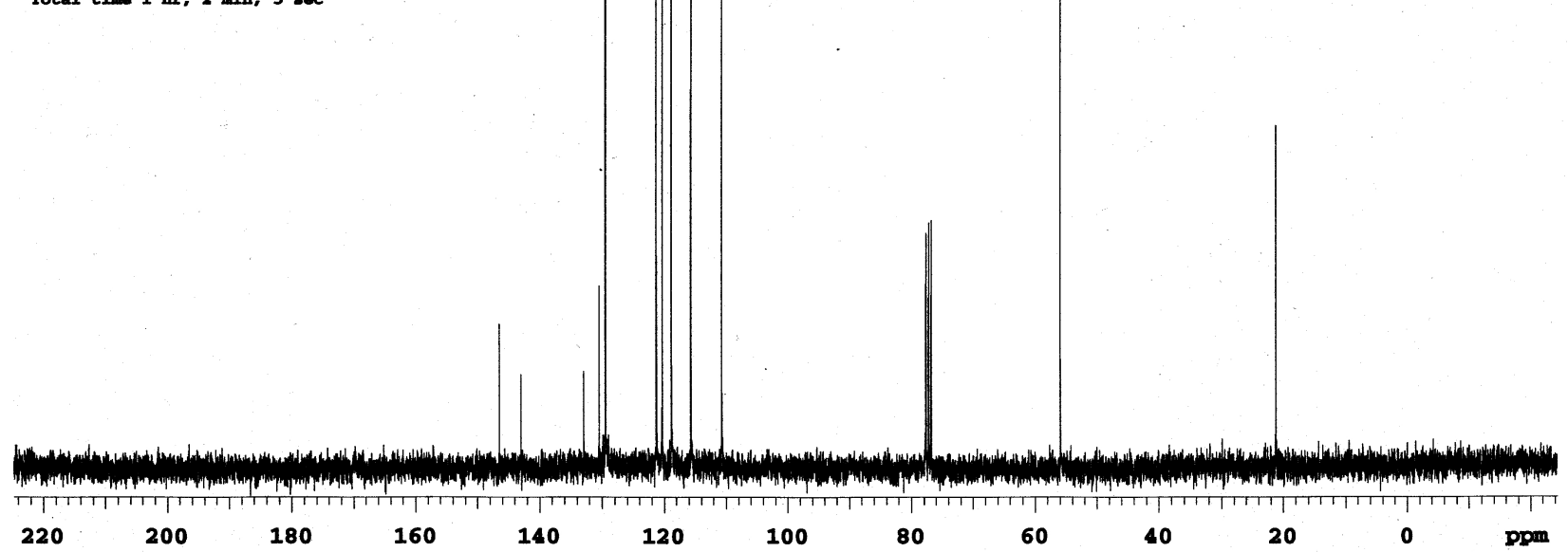


STANDARD 1H OBSERVE

Data Collected on:

pochacco-inova50

Archive directory:

/home/tquach/vnmrsys/dat

Sample directory:

File: TQ-05-79H

Pulse Sequence: s2pul

Solvent: $\operatorname{coc} 13$

Relax. delay $1.000 \mathrm{sec}$

Pulse 45.0 degrees

Acq. time 1.998 se

width $4500.5 \mathrm{~Hz}$

16 repetitions

OESERVE H1, 300
DATA PROCESSING

Line broadening $0.2 \mathrm{~Hz}$

FT size 32768

Total time 0 min

4b

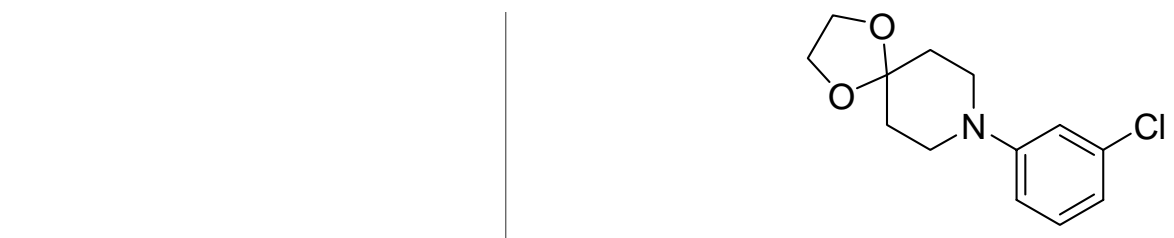

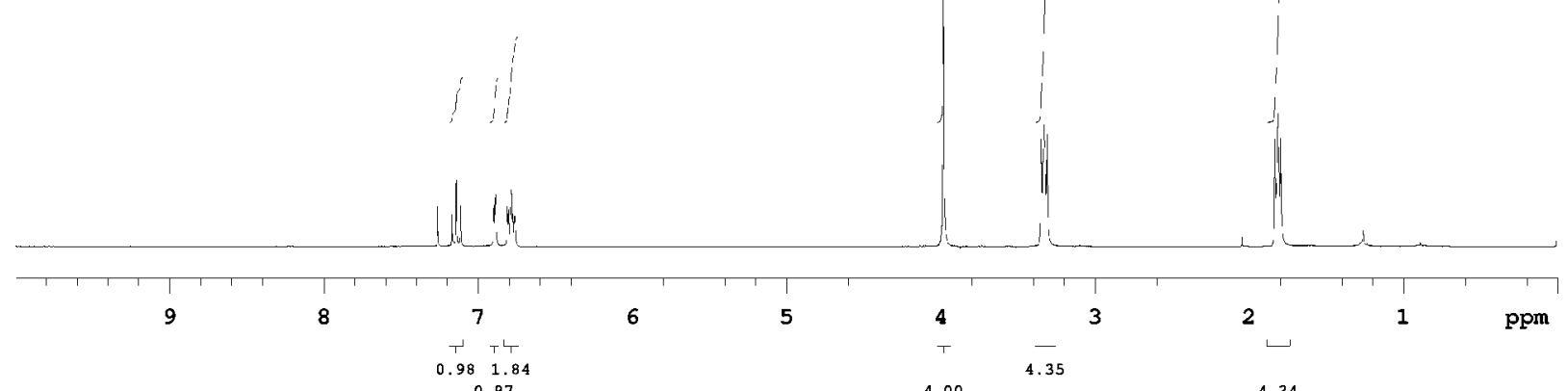

13C OBSERVE

Data collected on:

pochacco-inova500

Archive directory:

/tquach/vnmrsys/dat

Sample directory:

File: TQ-05-79C

Pulse Sequence: $s 2 \mathrm{pul}$

Solvent: $\mathrm{CDCl}_{3}$

Temp. $25.0 \mathrm{C} / 298.1 \mathrm{~K}$

Pulse 49.2 degrees

Acq. time $1.000 \mathrm{sec}$

width $18797.0 \mathrm{~Hz}$

344 repetitions

DECOUPLE H1, $300.0753189 \mathrm{MHz}$

Power $40 \mathrm{~dB}$

continuously on

DATA PROCESSING

Line broadening $1.0 \mathrm{~Hz}$

FT size 131072

Total time $1 \mathbf{h r}, 2$ min

\subsection{0}

4.34

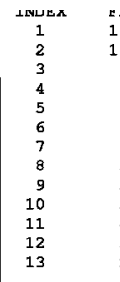

$\begin{array}{ll}11468.849 & 151.999 \\ 11468 & 151.90\end{array}$ $10188.468 \quad 135.030$ $9822.194 \quad 130.175$ $979.793 \quad 119.011$ $8633.597 \quad 114.423$ $8084.616 \quad 107.147$ $5857.430 \quad 77.630$ $525.019 \quad 77.200$ $5793.181 \quad 76.778$ $3572.879 \quad 47.352$

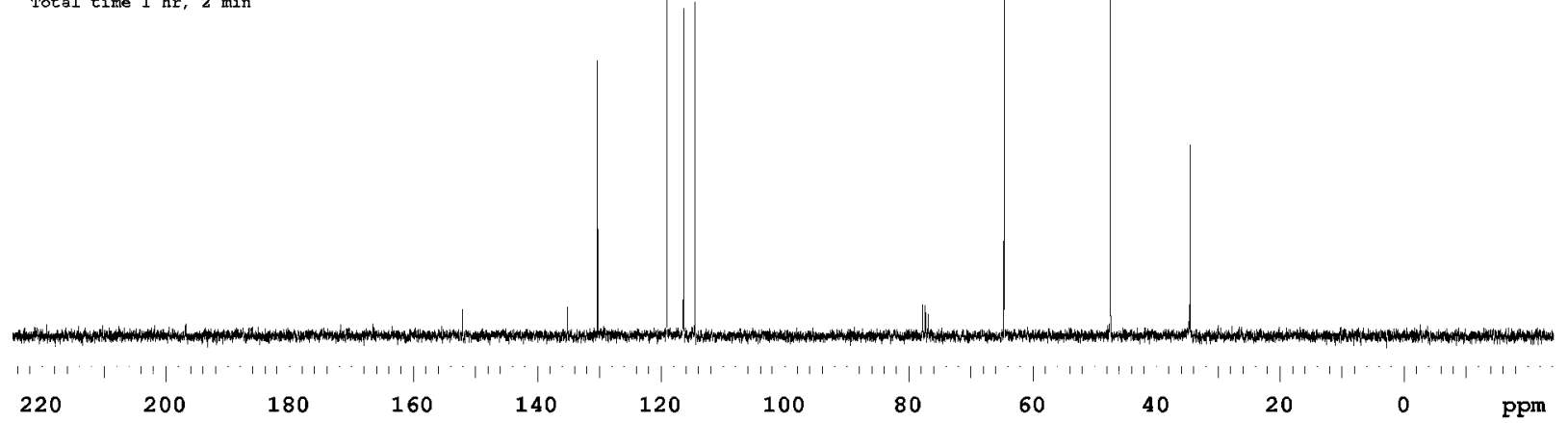


StANDARD 1H OBSERVE

Data collected on:

pochacco-inova50

Axchive directory:

/home/tquach/vnmrsys/dat

Sample directory:

File: TQ-05-82H

Pulse Sequence: $\mathbf{s 2 \mathrm { pul }}$

Solvent: $\operatorname{CDCl} 3$

Relax. delay $1.000 \mathrm{sec}$

Pulse 45.0 degrees

Acq. time 1.998 sec

width $4500.5 \mathrm{~Hz}$

16 repetitions

OBSERVE H1, 300.0738184 MHz

DATA EROCESSING

Line broadening $0.2 \mathrm{~Hz}$

FT size 32768

Total time $0 \mathrm{mi}$

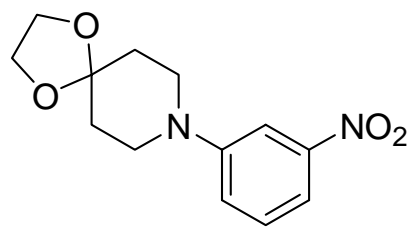

$4 c$

Data Collected on:

pochacco-inova 500

Archive directory:

/home/tquach/vnmrsys/dat

Sample directory:

File: $\mathrm{TQ}-05-82 \mathrm{C}$

Pulse Sequence: s2pul

Solvent: $\operatorname{CDCl} 3$

Pulse 28.6 degrees

Acq. time $1.815 \mathrm{sec}$

width $18761.7 \mathrm{~Hz}$

224 repetitions

OBSERVE C13， 75.4536142 MHz

DECOUPLE H1, 300.0753189 MP

Power $33 \mathrm{~dB}$

continuously on

WALTZ-16 modulated

Line broadening $1.0 \mathrm{~Hz}$

FT size 131072

Total time $36 \mathrm{~min}$

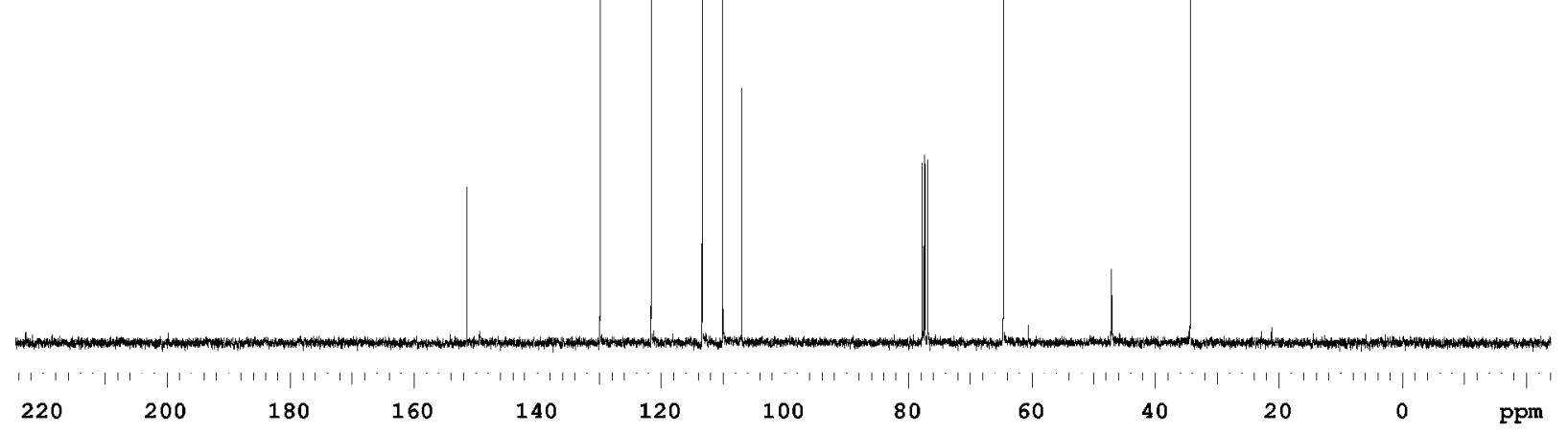


STANDARD 1H OBSERVE

Data collected on:

pochacco-inova500

Archive directory:

/home/tquach/vnmrsys/data

Sample directory:

File: TQ-05-81H

Pulse Sequence: $\mathbf{s 2 \mathrm { pul }}$

Solvent: $\operatorname{CDCl} 3$

Relax. delay $1.000 \mathrm{sec}$

Pulse 45.0 degrees

Acq. $t$ ime $1.998 \mathbf{s e c}$

width $4500.5 \mathrm{~Hz}$

16 repetitions

OBSERVE H1, 300.0738184 MHz

DATA PROCESSING

Line broadening $0.2 \mathrm{~Hz}$

FT size 32768

Total time $0 \mathrm{mi}$

Data collected on:

pochacco-inova 500

Archive directory:

/home/tquach/vn

Sample directory:

File: TQ-05-81C

Pulse Sequence: s2pul

Solvent: $\operatorname{CDCl} 3$

Pulse 28.6 degrees

Acq. time $1.815 \mathrm{sec}$

Width $18761.7 \mathrm{~Hz}$

128 repetitions

OBSERVE C13， $75.4536133 \mathrm{MHz}$

DECOUPLE H1, 300.0753189 MH

continuously on

WALT2-16 modulated

Line broadening 1.0

FT size 131072

Total time

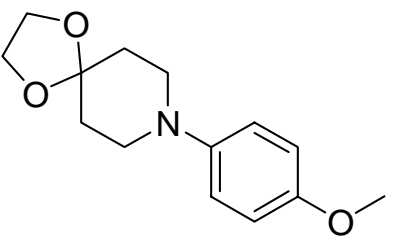

4d

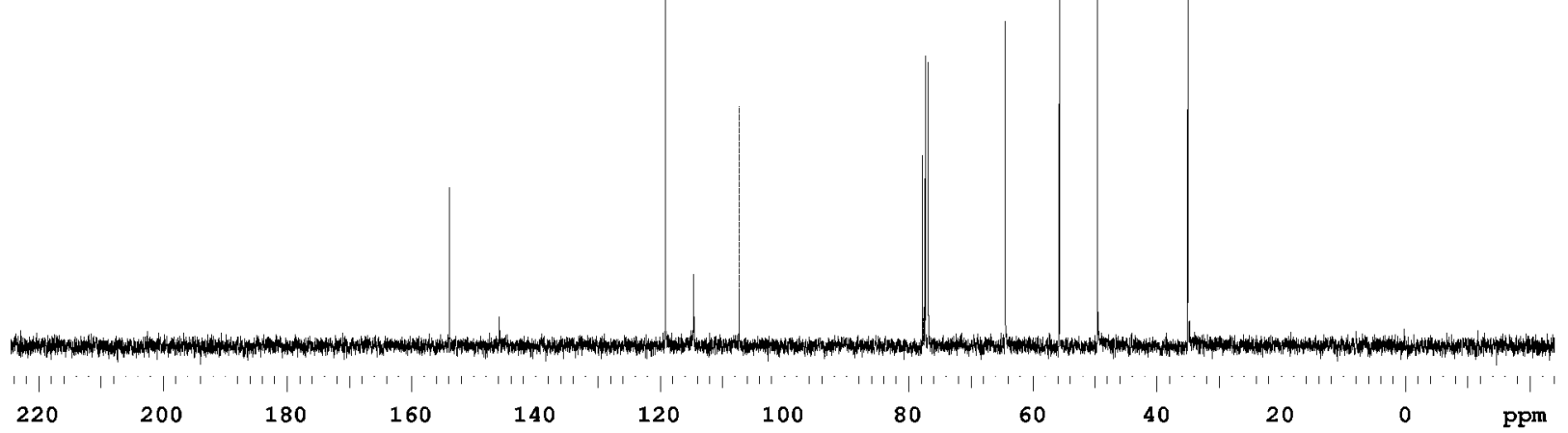


StANDARD 1H OBSERVE

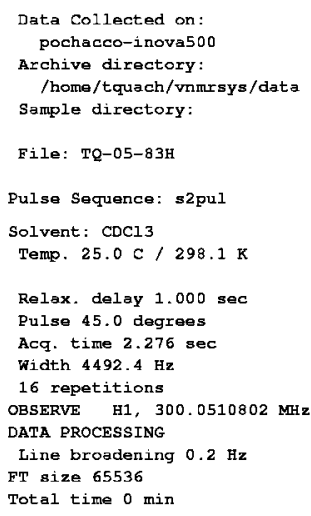

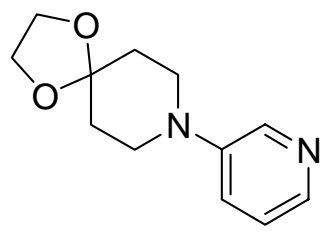

4e

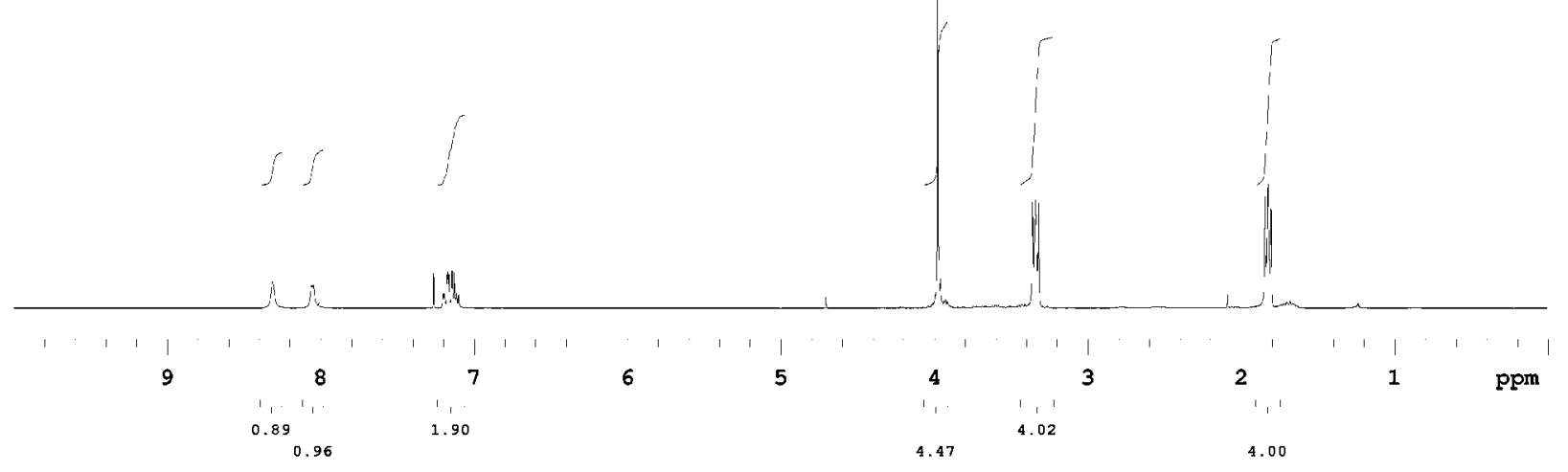

13C OBSERVE

Data Collected on:

pochacco-inova 50

Archive directory:

/home/tquach/vnmrsys/data

sample directory:

File: TQ-05-83C

Pulse Sequence: s2pul

Solvent: $\mathrm{CDC}_{13}$

Temp. $25.0 \mathrm{C} / 298.1 \mathrm{~K}$

Pulse 49.2 degrees

Acq. time $1.000 \mathrm{sec}$

width $18797.0 \mathrm{~Hz}$

1080 repetitions

OBSERVE $\mathrm{C} 13,75.4478913 \mathrm{MHz}$

DECOUPLE H1， $300.0525807 \mathrm{MHz}$

Power $40 \mathrm{~dB}$

continuously on

WALTZ-16 modulated

DATA PROCESSING

Iine broadening $1.0 \mathrm{~Hz}$

FT size 131072

Total time 0 min

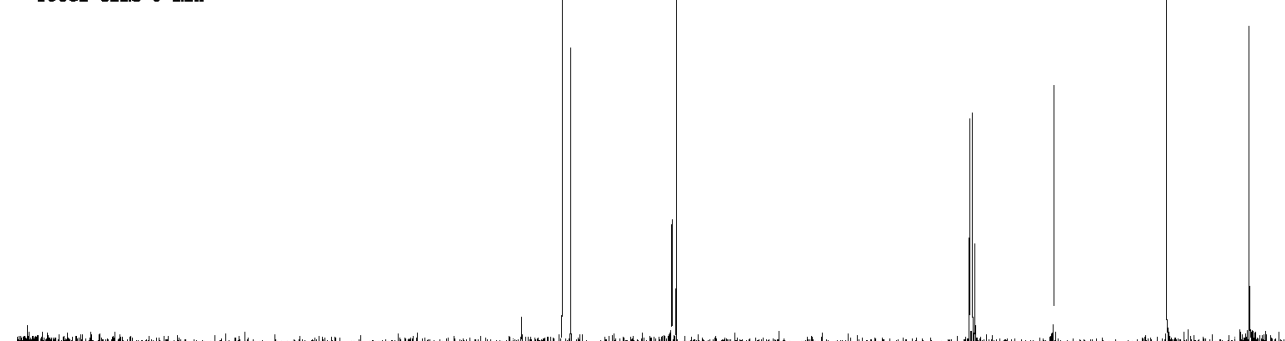

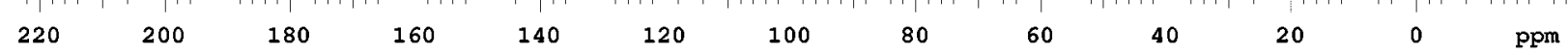


STANDARD 1H OBSERVE

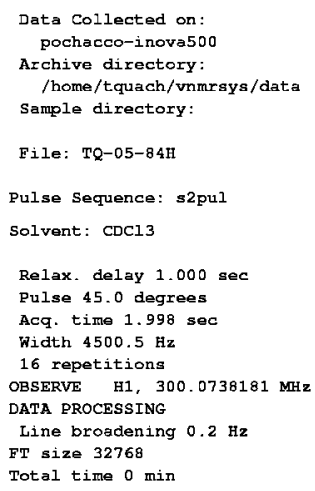

Relax delay 1.000 sec Pulse 45.0 degrees Acq. time $1.998 \mathrm{sec}$

Width $4500.5 \mathrm{~Hz}$

16 repetitions

OBSERVE H1, 300.0738181 MHE

DATA PROCESSING

Line broadening $0.2 \mathrm{~Hz}$

FT size

Total time $0 \mathrm{~min}$

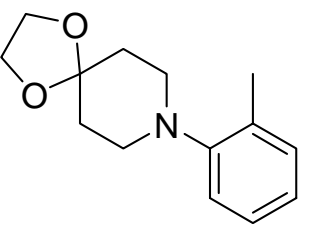

$4 f$

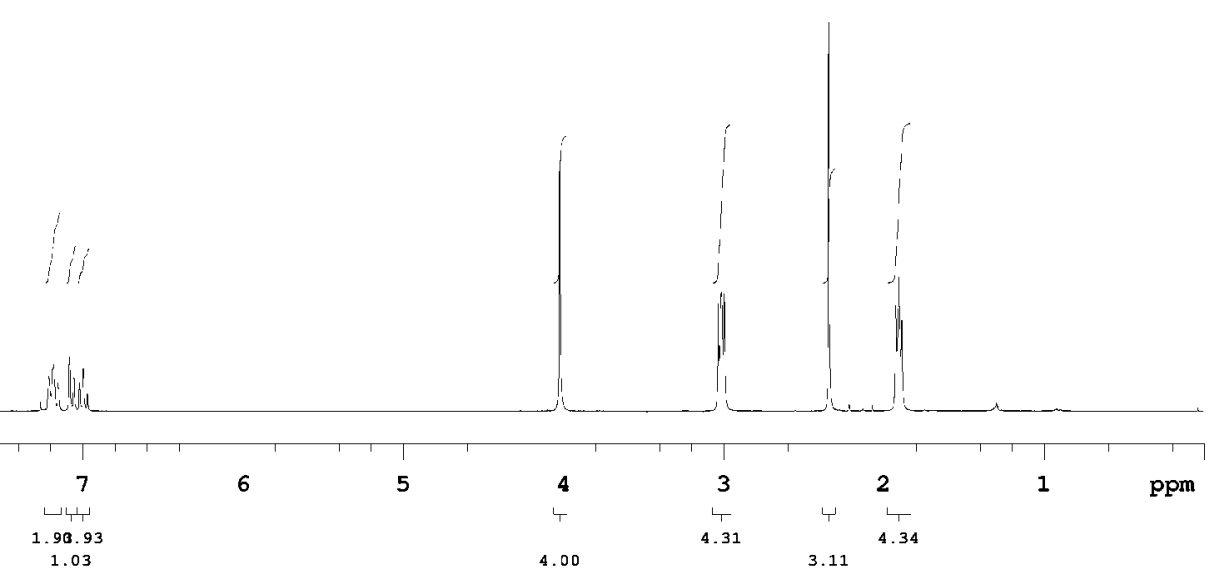

13C OBSERVE

Data Collected on:

pochacco-inova 500

Archive directory:

/home/tquach/vnmrsys/data

Sample directory:

File: TQ-05-84C

Pulse Sequence: s2pul

Solvent: $\operatorname{CDCl} 3$

Pulse 28.6 degrees

Acq. time 1.815 se

Width $18761.7 \mathrm{~Hz}$

176 repetitions

OBSERVE C13， 75.4536136 MH:

DECOUPLE H1, 300.0753189 MH

Power $33 \mathrm{~dB}$

continuously on

WAITZ-16 modulated

DATA EROCESSING

Line broadening $1.0 \mathrm{~Hz}$

FT size 131072

Total time $40 \mathrm{~min}$

4.00

3.11

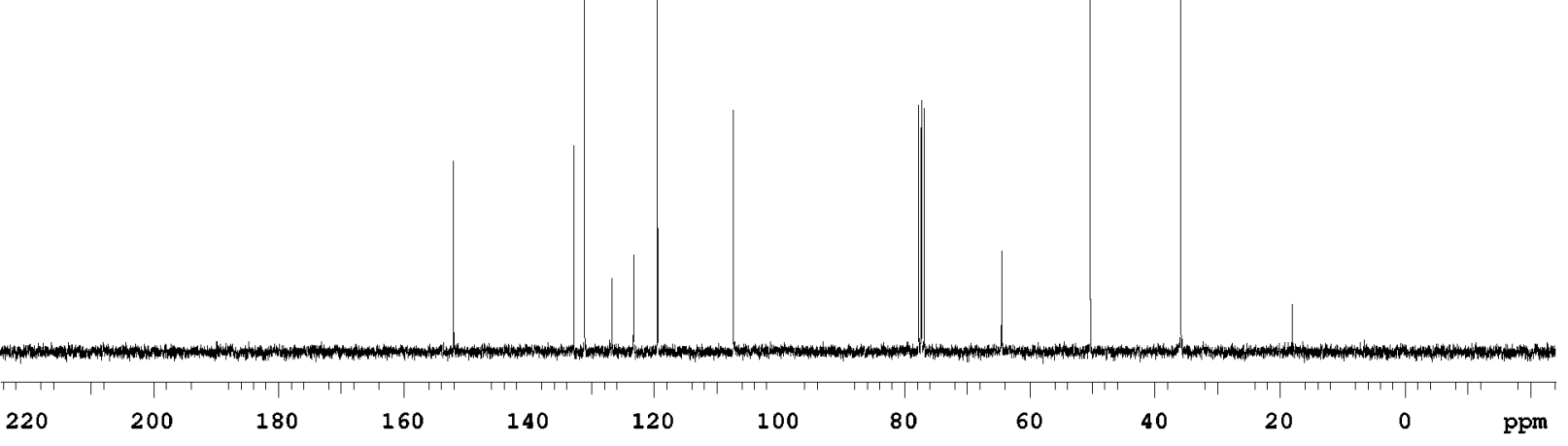

